\title{
Super-Electron Donors: Bis-pyridinylidene Formation by Base Treatment of Pyridinium Salts
}

\author{
John A. Murphy, ${ }^{* a}$ Jean Garnier, ${ }^{a}$ Stuart R. Park, ${ }^{a}$ Franziska Schoenebeck, ${ }^{a}$ \\ Sheng-ze Zhou, ${ }^{a}$ and Andrew T. Turner ${ }^{b}$ \\ ${ }^{a}$ WestCHEM, Department of Pure and Applied Chemistry, University of Strathclyde, 295 Cathedral Street, Glasgow G1 \\ 1XL, United Kingdom and ${ }^{\mathrm{b}}$ UM4 Main Lb Block Process R\&D, AstraZeneca, Hurdsfield Industrial Estate, Macclesfield \\ SK10 2NA, United Kingdom
}

\section{Materials and Methods}

Reactions were performed in flame-dried apparatus under a nitrogen or argon atmosphere using dry and deoxygenated solvents. Glove Box (System One Glove Box, Innovative Technology Inc., U.S.A.) was used to handle extremely airsensitive and moisture-sensitive reagents and reactions under a nitrogen atmosphere (oxygen and moisture levels were maintained at 0-2 ppm at all times). As required, organic solvents were dried and / or distilled prior to use. Tetrahydrofuran, dichloromethane, hexane, diethyl ether and toluene were dried and deoxygenated with a Pure-Solv 400 solvent purification system by Innovative Technology Inc., U.S.A. Anhydrous DMF was purchased commercially from Sigma-Aldrich Chemical Company.

Flash chromatography was performed using silica gel 60 (200-400 mesh). Thin layer chromatography (TLC) was performed using aluminium sheets of silica gel $60 \mathrm{~F}_{254}$, and plates were developed with aqueous potassium permanganate solution, methanolic phosphomolybdic acid $(10-20 \% \mathrm{w} / \mathrm{v})$, acidic $p$-anisaldehyde $(10 \% \mathrm{v} / \mathrm{v})$ or acidic methanolic vanillin solutions.

Gas Chromatography Mass Spectrometry (GC-MS) was performed on Thermo Finnigan PolarisQ Ion Trap Mass Spectrometer/Trace GC instrument with ZB-5 column (30 metres), at 1 $\mathrm{ml} / \mathrm{min}$ He gas flow rate and temperature range of 50 to $320^{\circ} \mathrm{C}$ with an increment of 10 to $20^{\circ} \mathrm{C} / \mathrm{min}$.

High and low resolution mass spectra were recorded at the EPSRC National Mass Spectrometry Service Centre, Swansea on a JLZX 102, VGZAB-E or a VG micromass instrument. The spectra were recorded using electron impact (EI), chemical ionization $(\mathrm{CI})$, fast atom bombardment $(\mathrm{FAB})$ or electrospray ionization (ESI) techniques as stated for each compound.

Proton NMR $\left({ }^{1} \mathrm{H}\right)$ spectra were recorded at $400 \mathrm{MHz}$ on a Bruker DPX 400 spectrometer. Carbon NMR $\left({ }^{13} \mathrm{C}\right)$ spectra were recorded at $100 \mathrm{MHz}$ on the same instrument. The chemical shifts ( $\delta$ ) are quoted in parts per million (ppm). Multiplicities are abbreviated as: s, singlet; d, doublet; t, triplet; q, quartet; m, multiplet. The coupling constants $(J)$ are measured in Hertz (Hz). Infra-red spectra were recorded on Perkin Elmer Spectrum One FT-IR spectrometer. 
Electrochemical experiments were performed inside a glove box under a nitrogen atmosphere at $21 \pm 1{ }^{\circ} \mathrm{C}$. Cyclic voltammograms were recorded using an Autolab PGSTAT30 potentiostat/galvanostat with a scan rate of $50 \mathrm{mV} / \mathrm{s}$. A three-electrode system was employed consisting of a platinum working electrode, a platinum wire auxiliary electrode and a $\mathrm{Ag} / \mathrm{AgCl} / \mathrm{KCl}$ (sat) double-junction electrode as the reference electrode. The supporting electrolyte, tetra- $n$ butylammonium hexafluorophosphate of $98 \%$ purity was purchased from Sigma-Aldrich Chemical Company and was dried in vacuo prior to use. Half-wave potentials $\left(\mathrm{E}_{1 / 2}\right)$ were calculated as an average of the cathodic and anodic peak potentials. The difference between anodic and cathodic peak potentials $(\mathrm{mV})$ is given in parenthesis. The irreversible (ir) peak potentials are denoted as $\mathrm{E}_{\mathrm{p}}$ and are given as the peak for the cathodic wave. Calibration was performed with ferrocene $\mathrm{E}_{1 / 2}(\mathrm{DMF})=0.5454 \mathrm{~V},(10.3526 \mathrm{mM}), \mathrm{Bu}_{4} \mathrm{NPF}_{6}(49.879 \mathrm{mM})$. Unless otherwise mentioned, the redox potentials for each of the compounds were reported as an average of five runs.

\section{Synthesis of donor 10}

Stage 1.

Synthesis of salt $7 \mathrm{~b} ; 1,3-b i s(N, N$-dimethyl-4-aminopyridinium)propane diiodide

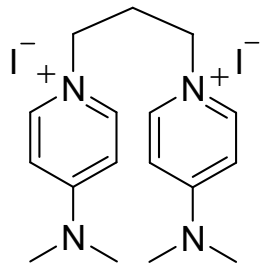

$7 \mathbf{b}$

A solution of 4-dimethylaminopyridine (4.58 g, $37.5 \mathrm{mmol}, 2.5$ eq.) and 1,3-diiodopropane (4.44 g, $15 \mathrm{mmol}, 1 \mathrm{eq}$.$) in$ acetonitrile $(100 \mathrm{ml})$ was stirred at reflux overnight, under an inert atmosphere. After cooling, the solid was filtered. Diethyl ether $(20 \mathrm{ml})$ was added to the filtered acetonitrile solution, precipitating more of the solid. The solid was washed with diethyl ether $(3 \mathrm{x} 30 \mathrm{ml})$ and dried under vacuum to give 1,3-bis $\left(N^{\prime}, N^{\prime}\right.$-dimethyl-4aminopyridinium)propane diiodide $7 \mathbf{b}(7.34 \mathrm{~g}, 90.6 \%)$ as a white powder; $\mathrm{mp} 280$ - $285^{\circ} \mathrm{C}$ (dec.); [Found: (ESI ${ }^{+}$) (M-

I) ${ }^{+}$413.1194. $\mathrm{C}_{17} \mathrm{H}_{26} \mathrm{I}_{2} \mathrm{~N}_{4}$ requires M-I, 413.1197]; $v_{\max }(\mathrm{KBr}) / \mathrm{cm}^{-1} 3005,1651,1574,1540,1404,1204,1185,1129$, 1067, 1036, 829, 805; ${ }^{1} \mathrm{H}-\mathrm{NMR}\left(400 \mathrm{MHz}, \mathrm{DMSO}-\mathrm{d}_{6}\right) \delta 2.36\left(2 \mathrm{H}\right.$, quintet, $\left.J=7.2 \mathrm{~Hz}, \mathrm{CH}_{2}\right), 3.20\left(12 \mathrm{H}, \mathrm{s}, \mathrm{CH}_{3}\right), 4.27$ $\left(4 \mathrm{H}, \mathrm{t}, J=7.2 \mathrm{~Hz}, \mathrm{NCH}_{2}\right), 7.05(4 \mathrm{H}, \mathrm{d}, J=7.7 \mathrm{~Hz}, \mathrm{ArH}), 8.30(4 \mathrm{H}, \mathrm{d}, J=7.7 \mathrm{~Hz}, \mathrm{ArH}) ;{ }^{13} \mathrm{C}$ NMR $(100 \mathrm{MHz}, \mathrm{DMSO}-$ $\left.\mathrm{d}_{6}\right) \delta 31.0\left(\mathrm{CH}_{2}\right), 39.8\left(4 \mathrm{CH}_{3}\right), 53.6\left(2 \mathrm{CH}_{2}\right), 107.8(2 \mathrm{CH}), 141.8(2 \mathrm{CH}), 155.8(2 \mathrm{C}) ; m / z\left(\mathrm{ESI}^{+}\right) 413\left[(\mathrm{M}-\mathrm{I})^{+}, 4 \%\right]$, $143(100), 96(10)$.

Stage 2

$N, N, N^{\prime}, N^{\prime}$-Tetramethyl-7,8-dihydro-6H-dipyrido[1,2-a;2',1'-c][1,4]diazepine-2,12-diamine 10<smiles></smiles> 
A mixture of 1,3-bis( $N, N$-dimethyl-4-aminopyridinium)propane diiodide $7 \mathbf{b}(8.10 \mathrm{~g}, 15 \mathrm{mmol}, 1$ eq.) and $\mathrm{NaH}(60 \%$ dispersed in mineral oil, $6 \mathrm{~g}, 150 \mathrm{mmol}, 10$ eq.) was placed under argon in a Schlenk flask equipped with a dry-ice cooler. The mixture was washed with hexane and dried under vacuum before being put back under argon. While stirring, ammonia gas was condensed into the flask $(70 \mathrm{ml})$, the mixture was kept under reflux for $4 \mathrm{~h}$ and then the ammonia was allowed to evaporate over $3 \mathrm{~h}$. Once the flask was at room temperature, still under argon, it was transferred to a glove box, where it was left overnight under vacuum. The solid was then extracted with diethyl ether, the solvent was removed by distillation and the residue dried under vacuum to give $N, N, N^{\prime}, N^{\prime}$-tetramethyl-7,8-dihydro6H-dipyrido [1,2-a;2',1'-c] [1,4] diazepine-2,12-diamine 10 (3.56 g, 83.4\%) as a purple-black, moisture-sensitive and oxygen-sensitive powder; ${ }^{1} \mathrm{H}$ NMR $\left(400 \mathrm{MHz}\right.$, Benzene- $\left.\mathrm{d}_{6}\right) \delta 1.00\left(2 \mathrm{H}\right.$, quintet, $\left.J=6.3 \mathrm{~Hz}, \mathrm{CH}_{2}\right), 2.46[12 \mathrm{H}, \mathrm{s}, 2$ $\left.\mathrm{N}\left(\mathrm{CH}_{3}\right)_{2}\right], 3.03\left(4 \mathrm{H}, \mathrm{t}, J=6.3 \mathrm{~Hz}, 2 \mathrm{NCH}_{2}\right), 4.91(2 \mathrm{H}, \mathrm{dd}, J=7.5,2.2 \mathrm{~Hz}, 2 \mathrm{ArH}), 5.14(2 \mathrm{H}, \mathrm{d}, J=2.2 \mathrm{~Hz}, 2 \mathrm{ArH})$, $5.64(2 \mathrm{H}, \mathrm{d}, J=7.5 \mathrm{~Hz}, 2 \mathrm{ArH}) ;{ }^{13} \mathrm{C}$ NMR $\left(100 \mathrm{MHz}\right.$, Benzene-d 6 ) $\delta 24.5\left(\mathrm{CH}_{2}\right), 40.8\left(4 \mathrm{CH}_{3}\right), 52.6\left(2 \mathrm{CH}_{2}\right), 95.8(2$ $\mathrm{CH}), 96.2(2 \mathrm{CH}), 116.0(2 \mathrm{C}), 138.7(2 \mathrm{CH}), 143.7$ (2 C). Cyclic voltammetry showed E1/2 $=-1.1340 \mathrm{~V} \pm 0.0116 \mathrm{~V}$.

\section{$N, N, N^{\prime}, N^{\prime}$-Tetramethyl-7,8-dihydro-6H-dipyrido[1,2a-2,1c][1,4]-diazepine-5,9-diium-2,12-diamine diiodide 11}

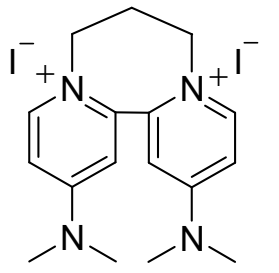

11

Under inert conditions, donor 10 (284 mg, $1 \mathrm{mmol}, 1$ eq.) was dissolved in dry and degassed acetonitrile (5 ml) and transferred onto iodine (280 $\mathrm{mg}, 1.1 \mathrm{mmol}, 1.1 \mathrm{eq}$.). After $15 \mathrm{~min}$, the mixture was filtered, and the solid washed thoroughly with diethyl ether and dried under vacuum to give $N, N, N^{\prime}, N^{\prime}$-tetramethyl-7,8-dihydro-6H-dipyrido[1,2a2,1c]-[1,4]-didiazepinium-2,12-diamine diiodide 11 (408 $\mathrm{mg}, 76 \%$ ) as a yellow powder; $\mathrm{mp} 182-186^{\circ} \mathrm{C}$ (dec); [Found: $\left(\mathrm{ESI}^{+}\right)(\mathrm{M}-\mathrm{I})^{+}$411.1043. $\mathrm{C}_{17} \mathrm{H}_{24} \mathrm{I}_{2} \mathrm{~N}_{4}$ requires M-I, 411.1040]; $v_{\max }(\mathrm{KBr}) / \mathrm{cm}^{-1} 3458,3389,3023,2986,1639$, 1571, 1531, 1428, 1404, 1371, 1319, 1260, 1180; ${ }^{1} \mathrm{H}$ NMR (400 MHz, DMSO-d $\left.{ }_{6}\right) \delta 2.40-2.43\left(2 \mathrm{H}, \mathrm{m}, \mathrm{CH}_{2}\right), 3.30$ $\left(6 \mathrm{H}, \mathrm{s}, 2 \mathrm{NCH}_{3}\right), 3.34\left(6 \mathrm{H}, \mathrm{s}, 2 \mathrm{NCH}_{3}\right), 3.92-4.00\left(2 \mathrm{H}, \mathrm{m}, 1 \mathrm{H}\right.$ of each $\left.\mathrm{NCH}_{2}\right), 4.53-4.57\left(2 \mathrm{H}, \mathrm{m}, 1 \mathrm{H}\right.$ of each $\left.\mathrm{NCH}_{2}\right)$, $7.34(2 \mathrm{H}, \mathrm{dd}, J=7.6,3.1 \mathrm{~Hz}, 2 \mathrm{ArH}), 7.40(2 \mathrm{H}, \mathrm{d}, J=3.1 \mathrm{~Hz}, 2 \mathrm{ArH}), 8.44(2 \mathrm{H}, \mathrm{d}, J=7.6 \mathrm{~Hz}, 2 \mathrm{ArH}) ;{ }^{13} \mathrm{C} \mathrm{NMR}(100$ MHz, DMSO-d $\left.\mathrm{d}_{6}\right) \delta 28.6\left(\mathrm{CH}_{2}\right), 40.3\left(2 \mathrm{CH}_{3}\right), 40.5\left(2 \mathrm{CH}_{3}\right), 51.0\left(2 \mathrm{CH}_{2}\right), 108.1(2 \mathrm{CH}), 111.8(2 \mathrm{CH}), 142.7(2 \mathrm{C})$, $143.3(2 \mathrm{CH}), 156.2$ (2 C); m/z (ESI $\left.{ }^{+}\right) 411$ [(M-I) $\left.{ }^{+}, 8 \%\right], 229$ (8), 383 (12), 243 (55), 227 (100), 200 (15), 179 (30), 163 (35), $142(20), 134(80), 112(70)$.

\section{General procedures for the use of electron donor 10:}

Procedure $\alpha$ :

In a moisture-free and oxygen-free inert atmosphere, the donor $10(128 \mathrm{mg}, 0.45 \mathrm{mmol}, 1.5 \mathrm{eq}$.$) was dissolved in$ degassed DMF ( $5 \mathrm{ml})$. This solution was directly pipetted onto the dry and degassed substrate $(0.3 \mathrm{mmol}, 1$ eq.). The mixture was left to stir overnight, then added to water $(75 \mathrm{ml})$, and extracted with diethyl ether $(50 \mathrm{ml}$ and $2 \times 25 \mathrm{ml})$. The combined organic layers were then washed with water $(2 \times 50 \mathrm{ml})$, brine $(50 \mathrm{ml})$ and dried over $\mathrm{Na}_{2} \mathrm{SO}_{4}$. The 
crude oil obtained after evaporation under reduced pressure was purified by column chromatography to give the corresponding products as reported.

Procedure $\beta$ :

Donor 10 (256 mg, $0.45 \mathrm{mmol}, 3$ eq.) was dissolved in degassed DMF (5 ml) in a glove-box. This solution was directly pipetted onto the dry and degassed selected substrate $(0.3 \mathrm{mmol}, 1 \mathrm{eq}$.). The mixture was left to stir overnight at $100^{\circ} \mathrm{C}$, then added to water $(75 \mathrm{ml})$, and extracted with diethyl ether $(50 \mathrm{ml}$ and $2 \times 25 \mathrm{ml})$. The combined organic layers were then washed with water $(2 \times 50 \mathrm{ml})$, brine $(50 \mathrm{ml})$ and dried over $\mathrm{Na}_{2} \mathrm{SO}_{4}$. The crude oil obtained after evaporation under reduced pressure was purified by column chromatography to give the corresponding products as reported.

Substrate Synthesis

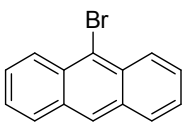

17

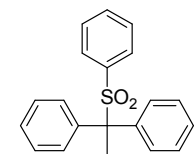

26

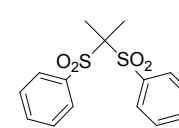

27

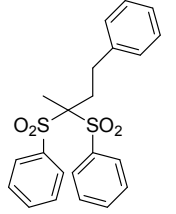

28

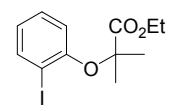

22

Substrate 17 was purchased and used as received from Sigma-Aldrich Co.

Substrates 26, $\mathbf{2 7}$ and $\mathbf{2 8}$ were synthesized as we previously described ${ }^{1}$, as was $\mathbf{2 2}^{2}$.

\section{1-Iodo-2-(3-phenylpropoxy)benzene 14}

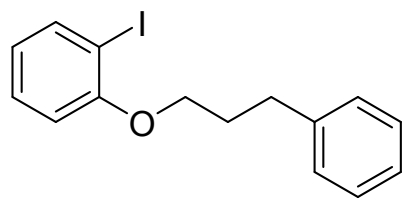

14

Potassium carbonate (7 g, $50 \mathrm{mmol}, 10$ eq.) was added to a solution of 2-iodophenol (1.1 $\mathrm{g}, 5 \mathrm{mmol}$, 1 eq.) and 1-bromo-3-phenylpropane ( $1.2 \mathrm{~g}, 6 \mathrm{mmol}, 1.2 \mathrm{eq}$.) in DMF $(50 \mathrm{ml})$. The suspension was stirred overnight, and then the DMF removed under reduced pressure. The solid residue was then dissolved in water $(75 \mathrm{ml})$ and extracted with diethyl ether $(50 \mathrm{ml}$ and $2 \times 25 \mathrm{ml})$. The combined organic layers were then washed with water $(2 \times 50$ $\mathrm{ml}$ ), brine $(50 \mathrm{ml})$ and dried over $\mathrm{Na}_{2} \mathrm{SO}_{4}$. The crude oil obtained after evaporation under reduced pressure was purified by column chromatography (gradient: $100 \%$ Pet. Ether to 20\% DCM / 80\% Pet. Ether) to give 1-iodo-2-(3phenylpropoxy)benzene 14 (1.66 g, 99\%) as a colourless oil; [Found: $\left(\mathrm{ESI}^{+}\right)\left(\mathrm{M}+\mathrm{NH}_{4}\right)^{+}$356.0510. $\mathrm{C}_{15} \mathrm{H}_{15} \mathrm{IO}$ requires $\left.\mathrm{M}+\mathrm{NH}_{4}, 356.0506\right] ; v_{\max }($ neat $) / \mathrm{cm}^{-1} 3060,3025,2945,2875,1581,1463,1275,1248,1049,1017,746,699 ;{ }^{1} \mathrm{H}-\mathrm{NMR}$ $\left(400 \mathrm{MHz}, \mathrm{CDCl}_{3}\right) \delta 2.14-2.21\left(2 \mathrm{H}, \mathrm{m}, \mathrm{CH}_{2}\right), 2.93\left(2 \mathrm{H}, \mathrm{t}, J=7.6 \mathrm{~Hz}, \mathrm{PhCH}_{2}\right), 4.02\left(2 \mathrm{H}, \mathrm{t}, J=6.1 \mathrm{~Hz}, \mathrm{OCH}_{2}\right), 6.70$ - $6.74(1 \mathrm{H}, \mathrm{ddd}, J=1.3,7.4,7.8, \mathrm{ArH}), 6.77(1 \mathrm{H}, \mathrm{dd}, J=1.2,8.2 \mathrm{~Hz}, \mathrm{ArH}), 7.20-7.33(6 \mathrm{H}, \mathrm{m}, 6 \mathrm{ArH}), 7.80(1 \mathrm{H}, \mathrm{dd}$, $J=1.6,7.8 \mathrm{~Hz}, \mathrm{ArH}) ;{ }^{13} \mathrm{C}-\mathrm{NMR}\left(100 \mathrm{MHz}, \mathrm{CDCl}_{3}\right) \delta 31.0\left(\mathrm{CH}_{2}\right), 32.3\left(\mathrm{CH}_{2}\right), 68.1\left(\mathrm{CH}_{2}\right), 86.9(\mathrm{C}), 112.3(\mathrm{CH}), 122.6$ $(\mathrm{CH}), 126.1(\mathrm{CH}), 128.6(\mathrm{CH}), 128.8(\mathrm{CH}), 129.6(\mathrm{CH}), 139.6(\mathrm{CH}), 141.7(\mathrm{C}), 157.7(\mathrm{C}) ; \mathrm{m} / z\left(\mathrm{CI}^{+}\right) 356\left[\left(\mathrm{M}^{+} \mathrm{NH}_{4}\right)^{+}\right.$, 100\%], $338\left(\mathrm{M}^{+}, 10 \%\right), 230(87), 228(23), 212(13)$. 


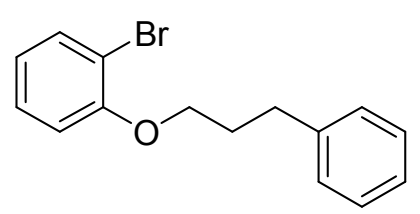

15

Following the same synthesis as for 14, ether 15 was prepared starting from 2-bromophenol ( $875 \mathrm{mg}, 5.1 \mathrm{mmol}, 1$ eq.). 1-Bromo-2-(3-phenylpropoxy)benzene (15) $(1.42 \mathrm{~g}, 96 \%)$ was obtained as a colourless oil; [Found: $\left(\mathrm{ESI}^{+}\right)\left(\mathrm{M}^{+} \mathrm{NH}_{4}\right)^{+}$ 308.0646. $\mathrm{C}_{15} \mathrm{H}_{15} \mathrm{BrO}$ requires $\left.\mathrm{M}+\mathrm{NH}_{4}, 308.0645\right]$; $v_{\max }($ neat $) / \mathrm{cm}^{-1}$ 3062, 3026, 2949, 2876, 1587, 1482, 1389, 1277 , 1249, 1051, 1031, 746, 700; ${ }^{1} \mathrm{H}-\mathrm{NMR}\left(400 \mathrm{MHz}, \mathrm{CDCl}_{3}\right) \delta 2.19-2.27\left(2 \mathrm{H}, \mathrm{m}, \mathrm{CH}_{2}\right), 2.97\left(2 \mathrm{H}, \mathrm{t}, J=7.6 \mathrm{~Hz}, \mathrm{PhCH}_{2}\right)$, $4.06\left(2 \mathrm{H}, \mathrm{t}, J=6.2 \mathrm{~Hz}, \mathrm{OCH}_{2}\right), 6.88-6.91(2 \mathrm{H}, \mathrm{m}, 2 \mathrm{ArH}), 7.27-7.40(6 \mathrm{H}, \mathrm{m}, 6 \mathrm{ArH}), 7.64(1 \mathrm{H}, \mathrm{dd}, J=1.6,8.2 \mathrm{~Hz}$, ArH); ${ }^{13} \mathrm{C}-\mathrm{NMR}\left(100 \mathrm{MHz}, \mathrm{CDCl}_{3}\right) \delta 30.9\left(\mathrm{CH}_{2}\right), 32.1\left(\mathrm{CH}_{2}\right), 68.0\left(\mathrm{CH}_{2}\right), 112.5(\mathrm{C}), 113.4(\mathrm{CH}), 121.9(\mathrm{CH}), 126.1$ $(\mathrm{CH}), 128.6(\mathrm{CH}), 128.7(\mathrm{CH}), 133.5(\mathrm{CH}), 141.6(\mathrm{C}), 155.5(\mathrm{C}) ; \mathrm{m} / z\left(\mathrm{CI}^{+}\right) 308\left[\left(\mathrm{M}^{+} \mathrm{NH}_{4}\right)^{+}, 100 \%\right], 290\left(\mathrm{M}^{+}, 10 \%\right)$, 230 (12), 212 (4), 118 (15), 108 (18), 91 (25).

\section{2-Iodo-1,3,5-tri-tert-butylbenzene $19^{3}$}

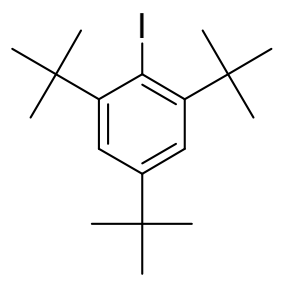

19

A solution of $n$-butyllithium in hexane $(2.52 \mathrm{M}, 2.50 \mathrm{ml}, 6.3 \mathrm{mmol}, 1.26 \mathrm{eq}$.) was added dropwise under an inert atmosphere to a solution of 2-bromo-1,3,5-tri-tert-butylbenzene (1.63 g, $5 \mathrm{mmol}, 1 \mathrm{eq}$.) in dry ether (40 ml). The solution was then refluxed for $1 \mathrm{~h}$, and then cooled to $0^{\circ} \mathrm{C}$. A solution of iodine (1.6 g, $6.3 \mathrm{mmol}, 1.26 \mathrm{eq}$.) in diethyl ether $(15 \mathrm{ml})$ was added over $20 \mathrm{~min}$. The resulting solution was brought to room temperature and stirred for $30 \mathrm{~min}$. Extraction of the products was performed with diethyl ether $(75+2 \times 25 \mathrm{ml})$ and saturated aqueous sodium sulfite (75 $\mathrm{ml})$. The combined organic layers were then washed with water $(2 \times 50 \mathrm{ml})$ and dried over $\mathrm{Na}_{2} \mathrm{SO}_{4}$. The yellow solid obtained after evaporation was purified by column chromatography (hexane), where fractions were tested by NMR to determine relative content of $\mathbf{1 9}$ and $\mathbf{2 0}$. Selection of the fraction containing pure $\mathbf{1 9}$ provided 2-iodo-1,3,5-tri-tertbutylbenzene (19) $(729 \mathrm{mg}, 39 \%)$ as a white amorphous solid; mp: $155-165^{\circ} \mathrm{C}$ (lit. $\left.{ }^{3} 175.7-177.0^{\circ} \mathrm{C}\right)$; [Found: $\left(\mathrm{EI}^{+}\right.$) $\left(\mathrm{M}^{+}\right)$372.1305. $\mathrm{C}_{18} \mathrm{H}_{29} \mathrm{I}$ requires $\mathrm{M}^{+}$, 372.1308]; $v_{\max }(\mathrm{KBr}) / \mathrm{cm}^{-1} 3008,2959,2867,1762,1590,1554,1474,1459$, 1411, 1394, 1383, 1362, 1237, 1213, 1190, 1131, 994, 876; ${ }^{1} \mathrm{H}-\mathrm{NMR}\left(400 \mathrm{MHz}, \mathrm{CDCl}_{3}\right) \delta 1.33$ (9H, s, p-tert-butyl), $1.68\left(18 \mathrm{H}, \mathrm{s}, 2\right.$ o-tert-butyl), $7.42(2 \mathrm{H}, \mathrm{s}, 2 \mathrm{ArH}) ;{ }^{13} \mathrm{C}-\mathrm{NMR}\left(100 \mathrm{MHz}, \mathrm{CDCl}_{3}\right) \delta 31.5\left(\mathrm{CH}_{3}\right), 31.9\left(\mathrm{CH}_{3}\right), 35.1(\mathrm{C})$, $39.6(\mathrm{C}), 95.8(\mathrm{C}), 123.8(\mathrm{CH}), 149.4(\mathrm{C}), 151.7$ (C); m/z (EI $\left.{ }^{+}\right) 372.2\left(\mathrm{M}^{+}, 17 \%\right), 357.2$ (7), 231.2 (12), 215.2 (5), 203.2 (8), 91.0 (16), $86.0(15), 83.9$ (22), 57.1 (100). 


\section{(3-Phenylpropoxy)benzene 16}

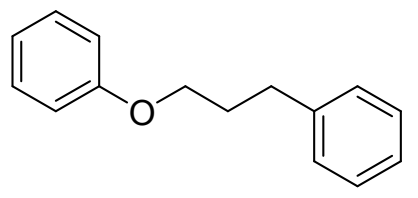

16

Application of procedure $\alpha$ to substrate $14(102 \mathrm{mg}, 0.302 \mathrm{mmol})$ and of procedure $\beta$ to substrate $15(92 \mathrm{mg}, 0.316$ $\mathrm{mmol}$ ), followed by column chromatography (hexane) provided (3-phenylpropoxy)benzene ${ }^{4}$ (16) as a colourless oil (59 mg, $0.278 \mathrm{mmol}, 92 \%$, respectively $55 \mathrm{mg}, 0.259 \mathrm{mmol}, 82 \%$ ); [Found: $\left(\mathrm{ESI}^{+}\right)\left(\mathrm{M}^{+} \mathrm{NH}_{4}\right)^{+}, 230.1538 . \mathrm{C}_{15} \mathrm{H}_{16} \mathrm{O}$ requires $\left.\mathrm{M}+\mathrm{NH}_{4}, 230.1539\right]$; $v_{\max }($ neat $) / \mathrm{cm}^{-1}$ 3062, 3027, 2946, 2870, 1600, 1497, 1245, 1038, 751; ${ }^{1} \mathrm{H}-\mathrm{NMR}$ (400 $\left.\mathrm{MHz}, \mathrm{CDCl}_{3}\right) \delta 2.15\left(2 \mathrm{H}, \mathrm{m}, \mathrm{CH}_{2}\right), 2.86\left(2 \mathrm{H}, \mathrm{t}, J=7.5 \mathrm{~Hz}, \mathrm{PhCH}_{2}\right), 4.01\left(2 \mathrm{H}, \mathrm{t}, J=6.3 \mathrm{~Hz}, \mathrm{OCH}_{2}\right), 6.93-7.00(3 \mathrm{H}$, $\mathrm{m}, \mathrm{ArH}), 7.22-7.35(7 \mathrm{H}, \mathrm{m}, \mathrm{ArH}) ;{ }^{13} \mathrm{C}-\mathrm{NMR}\left(100 \mathrm{MHz}, \mathrm{CDCl}_{3}\right) \delta 31.1\left(\mathrm{CH}_{2}\right), 32.4\left(\mathrm{CH}_{2}\right), 67.0\left(\mathrm{CH}_{2}\right), 114.8(\mathrm{CH})$, $120.8(\mathrm{CH}), 126.1(\mathrm{CH}), 128.6(\mathrm{CH}), 128.7(\mathrm{CH}), 129.6(\mathrm{CH}), 141.8(\mathrm{C}), 159.3(\mathrm{C}) ; \mathrm{m} / z\left(\mathrm{CI}^{+}\right) 230\left[\left(\mathrm{M}^{+} \mathrm{NH}_{4}\right)^{+}, 100 \%\right]$, $212\left(\mathrm{M}^{+}, 20 \%\right), 118(10), 108(13), 91(22)$.

\section{Anthracene 18}

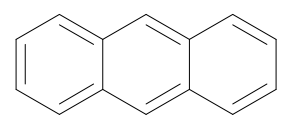

18

As product of procedure $\alpha$ applied to substrate $17(77 \mathrm{mg}, 0.299 \mathrm{mmol})$ followed by column chromatography (toluene), anthracene 18 was recovered as white slates (51 mg, $0.286 \mathrm{mmol}, 96 \%)$; mp: $205-210^{\circ} \mathrm{C}$ (lit: 205 $\left.210^{\circ} \mathrm{C}\right) ; v_{\max }(\mathrm{KBr}) / \mathrm{cm}^{-1} \quad 3048,1784,1620,1533,1448,1316,1272,1146,997,956,884,726 ;{ }^{1} \mathrm{H}-\mathrm{NMR}(400 \mathrm{MHz}$, $\left.\mathrm{CDCl}_{3}\right) \delta 7.47-7.51(4 \mathrm{H}, \mathrm{m}, 4 \mathrm{ArH}), 8.02-8.04(4 \mathrm{H}, \mathrm{m}, 4 \mathrm{ArH}), 8.45(2 \mathrm{H}, \mathrm{s}, 2 \mathrm{ArH}) ;{ }^{13} \mathrm{C}-\mathrm{NMR}\left(100 \mathrm{MHz}, \mathrm{CDCl}_{3}\right) \delta$ $125.6(\mathrm{CH}), 126.4(\mathrm{CH}), 128.4(\mathrm{CH}), 132.0(\mathrm{C})$. Analyses were consistent with the corresponding analyses performed on the commercial product (Aldrich).

\section{1,3,5-tri-tert-butylbenzene 20}

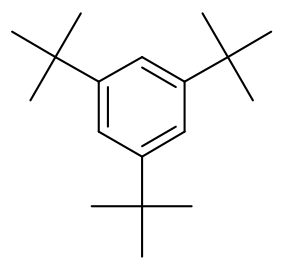

20

Application of procedure $\alpha$ to substrate $19(113 \mathrm{mg}, 0.303 \mathrm{mmol})$ followed by filtration on column chromatography (hexane) provided 1,3,5-tri-tert-butylbenzene ${ }^{6}(\mathbf{2 0})$ as a white amorphous solid (71 $\left.\mathrm{mg}, 0.288 \mathrm{mmol}, 95 \%\right)$; $\mathrm{mp}: 70$ $72^{\circ} \mathrm{C}\left(\mathrm{lit}^{6}: \mathrm{T0}-71^{\circ} \mathrm{C}\right) ; v_{\max }(\mathrm{KBr}) / \mathrm{cm}^{-1} 3077,2963,2902,2866,1781,1761,1598,1474,1449,1391,1362,1249,1202$, 
935, 900, 873, 714; ${ }^{1} \mathrm{H}-\mathrm{NMR}$ [400 MHz, $\left(\mathrm{CD}_{3}\right)_{2} \mathrm{CO}$ ] $\delta 1.32(27 \mathrm{H}, \mathrm{s}, 3$ tri-tert-butyl), 7.29 (3H, s, $3 \mathrm{ArH}) ;{ }^{13} \mathrm{C}-\mathrm{NMR}$ [100 MHz, $\left.\left(\mathrm{CD}_{3}\right)_{2} \mathrm{CO}\right] \delta 32.0\left(\mathrm{CH}_{3}\right), 35.6(\mathrm{C}), 120.1(\mathrm{CH}), 150.7(\mathrm{C}) . \mathrm{m} / z\left(\mathrm{EI}^{+}\right) 246.0\left(\mathrm{M}^{+}, 16 \%\right), 231.1(87), 175.1$ (12), $57.1(100)$

\section{$\underline{\text { 2,4,6-Tri-tertbutylbromobenzene and 2,4,6-Tri-tertbutyliodobenzene reduction in presence of } \mathrm{D}_{2}} \underline{\mathrm{O}}$}<smiles>CC(C)(C)c1cc(C(C)(C)C)c(I)c(C(C)(C)C)c1</smiles>

19
15: 1.5 eq. $10, \mathrm{rt}$

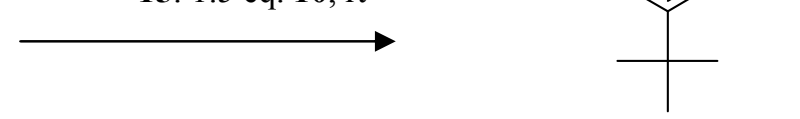

20

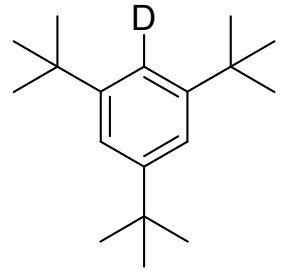

21

Application of procedure $\alpha$ to substrate 19, to which 20 eq. of deuterated water had been added, followed by filtration on column chromatography (hexane) provided a mixture of 1,3,5-tri-tert-butylbenzene (20) and 1-deutero-2,4,6-tritert-butylbenzene (21), as a white solid (70 mg). ${ }^{1} \mathrm{H}-\mathrm{NMR}\left[400 \mathrm{MHz},\left(\mathrm{CD}_{3}\right)_{2} \mathrm{CO}\right] \delta 1.33(27.00 \mathrm{H}, \mathrm{s}, 3$ tri-tert-butyl), 7.29 (2.04 H, s, aromatic hydrogens); ${ }^{2} \mathrm{H}-\mathrm{NMR}\left[61.4 \mathrm{MHz},\left(\mathrm{CD}_{3}\right)_{2} \mathrm{CO}\right] \delta 7.32$ (Ar-D); ${ }^{13} \mathrm{C}-\mathrm{NMR}\left(400 \mathrm{MHz}, \mathrm{CDCl}_{3}\right) \delta$ $32.1\left(\mathrm{CH}_{3}\right), 35.6(\mathrm{C}), 120.1(\mathrm{CH}), 150.6(\mathrm{C}) .150 .7(\mathrm{C})$.

GC-MS measurement provided a unique peak for the mixture, which has a consistent retention time with a previously injected $\mathbf{2 0}$ reference.

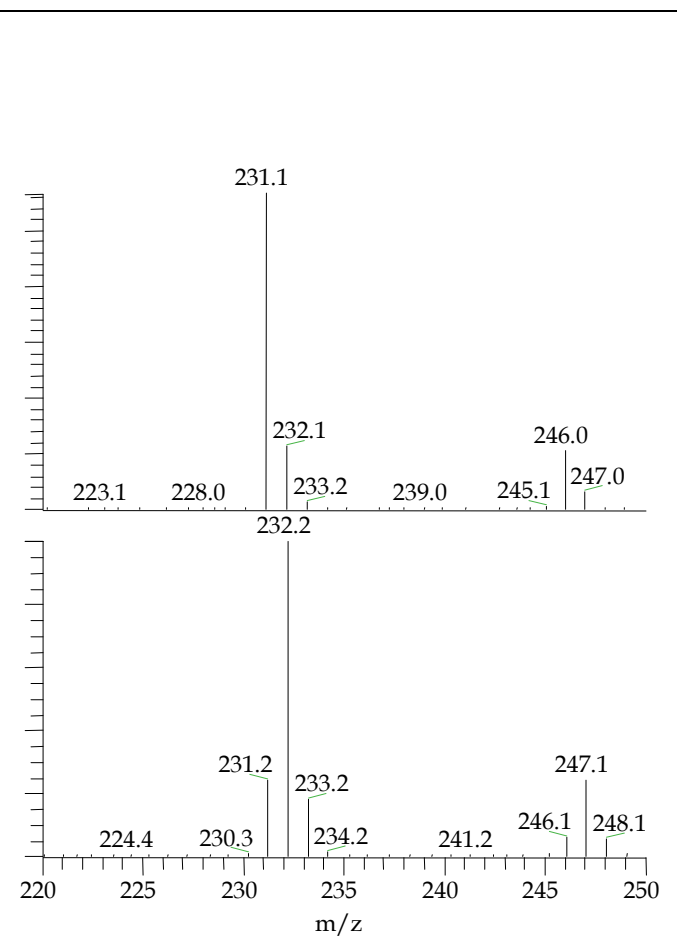

\begin{tabular}{|c|c|}
\hline \multicolumn{2}{|c|}{20 reference: } \\
\hline Peak & $\begin{array}{l}\text { Intensity } \\
\text { (relative) }\end{array}$ \\
\hline 231.1 & 87.5 \\
\hline 232.1 & 17.3 \\
\hline 233.2 & 1.91 \\
\hline 246.0 & 16.2 \\
\hline 247.0 & 4.64 \\
\hline 248.0 & 0.40 \\
\hline \multicolumn{2}{|c|}{$20+21$ Mixture reference: } \\
\hline Peak & $\begin{array}{l}\text { Intensity } \\
\text { (relative) }\end{array}$ \\
\hline 231.2 & 24.5 \\
\hline 232.2 & 100 \\
\hline 233.2 & 17.7 \\
\hline 246.1 & 5.70 \\
\hline 247.1 & 23.8 \\
\hline 248.1 & 5.32 \\
\hline
\end{tabular}

Based on the peak relative intensities above, the ratio 20:21 in the mixture was determined to be $\sim 1: 4$. 


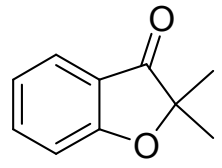

23

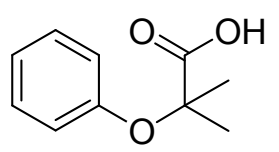

24

Application of procedure $\alpha$ with a 2-fold scale-up to substrate 22 (200 mg, $0.6 \mathrm{mmol}$ ) followed by purification on column chromatography (1:1 Pet. ether/DCM) provided an inseparable mixture (110 mg) of 23 and ethyl 2-methyl-2phenoxypropanoate 25. This mixture was then hydrolyzed overnight in a solution of $\mathrm{KOH}(40 \mathrm{mg})$ in water and methanol $(1: 3$ ratio, $2 \mathrm{ml})$ at $50^{\circ} \mathrm{C}$. Isolation of 23 by extraction of 24 was performed using ether (75 ml) and a sodium hydroxide solution $(1 \mathrm{M}, 50+25 \mathrm{ml})$, followed by drying of the organic phase $\left(\mathrm{MgSO}_{4}\right)$, evaporation under vacuum and column chromatography (1:1 Pet. ether/DCM). Ketone 23 was obtained as a colourless oil (81 mg, $0.5 \mathrm{mmol}$, $83 \%$ ), with data identical to an authentic sample. Crude 24 was obtained after acidification and extraction of the aqueous phase by ether, $\mathrm{MgSO}_{4}$ drying of the organic phase and evaporation if the solvent under reduced pressure. Column chromatography (gradient DCM/AcOEt) provided the 2-methyl-2-phenoxypropionic acid 24 as a lightyellow powder $(9 \mathrm{mg}, 0.05 \mathrm{mmol}, 8.3 \%)$; mp: $95-96^{\circ} \mathrm{C}$ (lit. $\left.{ }^{7} 99-100^{\circ} \mathrm{C}\right)$; [Found: $\left(\mathrm{ESI}^{+}\right)\left(\mathrm{M}^{+} \mathrm{NH}_{4}\right)^{+}, 198.1125$. $\mathrm{C}_{10} \mathrm{H}_{12} \mathrm{O}_{3}$ requires $\left.\mathrm{M}+\mathrm{NH}_{4}, 198.1125\right] ; v_{\max }$ (neat)/cm $\mathrm{cm}^{-1}$ 3012, 3001, 2916, 2690, 2569, 1697, 1597, 1489, 1380, 1295, 1241, 1202, 1164, 1152, 1082, 1029, 976, 932, 812, 756; ${ }^{1} \mathrm{H}-\mathrm{NMR}\left(400 \mathrm{MHz}, \mathrm{CDCl}_{3}\right) \delta 1.62(6 \mathrm{H}, \mathrm{s}, 2 \mathrm{CH}), 6.96(2 \mathrm{H}$, $\mathrm{d}, J=7.8 \mathrm{~Hz}, 2 \mathrm{ArH}), 7.08(1 \mathrm{H}, \mathrm{t}, J=7.4 \mathrm{~Hz}, \mathrm{ArH}), 7.27-7.32(2 \mathrm{H}, \mathrm{m}, 2 \mathrm{ArH}) ;{ }^{13} \mathrm{C}-\mathrm{NMR}\left(100 \mathrm{MHz}^{\mathrm{CDCl}}\right)_{3} \delta 25.3$ $\left(\mathrm{CH}_{3}\right), 79.8(\mathrm{C}), 120.8(\mathrm{CH}), 123.5(\mathrm{CH}), 129.6(\mathrm{CH}), 154.5(\mathrm{C}), 178.5(\mathrm{C}) ; \mathrm{m} / z\left(\mathrm{EI}^{+}\right) 181\left((\mathrm{M}+\mathrm{H})^{+}, 5 \%\right), 134(8), 94$ (100).

\section{1,1-diphenylethane 29}

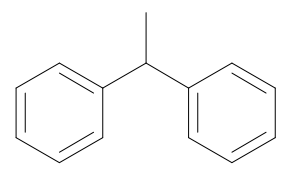

29

As product of procedure $\beta$ applied to substrate 26 (96 mg, $0.298 \mathrm{mmol}$ ), followed by column chromatography (Pet. ether), compound 29 was recovered as a colourless oil (53 mg, $0.291 \mathrm{mmol}, 98 \%$ ), which was consistent in ${ }^{1} \mathrm{H}-\mathrm{NMR}$ and ${ }^{13} \mathrm{C}-\mathrm{NMR}$ with previous data. ${ }^{1}$

\section{Isopropylsulfonylbenzene 30}

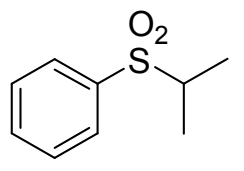


As product of procedure $\beta$ applied to substrate 27 (97 mg, $0.300 \mathrm{mmol}$ ) followed by column chromatography (Pet. ether), isopropylsulfonylbenzene 30 was recovered as a colourless oil $(53 \mathrm{mg}, 0.288 \mathrm{mmol}, 96 \%){ }^{1} \mathrm{H}$-NMR and ${ }^{13} \mathrm{C}$ NMR agreed with prior data. ${ }^{1}$

\section{1-Methyl-3-phenylpropyl phenyl sulfone 31}

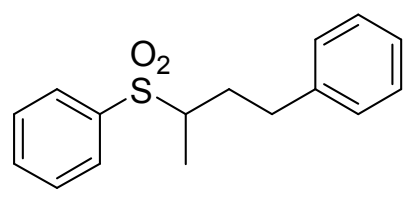

31

As product of procedure $\beta$ applied to substrate 28 (124 $\mathrm{mg}, 0.299 \mathrm{mmol})$ followed by column chromatography (Pet. ether), 1-methyl-3-phenylpropyl phenyl sulfone 31 was recovered as a colourless oil (81 mg, $0.295 \mathrm{mmol}, 99 \%) .{ }^{1} \mathrm{H}-$ NMR and ${ }^{13} \mathrm{C}$-NMR were consistent with prior data. ${ }^{1}$

\section{References.}

1. Schoenebeck, F.; Murphy, J. A.; Zhou, S.-Z.; Uenoyama, Y.; Miclo, Y.; Tuttle, T. J. Am Chem. Soc. 2007, 129, 13368-13369.

2. Murphy, J. A.; Zhou, S.-Z.; Thomson, D. W.; Schoenebeck, F.; Mohan, M.; Park, S. R.; Tuttle, T. ;

Berlouis, L. E. A. Angew. Chem. Int. Ed. 2007, 46, 5178-5183.

3. Zimmerman, H. E.; Dodd, J. R. J. Am. Chem. Soc. 1970, 92, 6507-6515.

4 Quach, T. D.; Batey, R. A. Org. Lett. 2003, 5, 1381-1384.

5 Paquette, L. A.; Browne, A. R.; Chamot, E.; Blount, J. F. J. Am. Chem. Soc. 1980, 102 , 643-651.

6. Frampton, M. J.; Akdas, H. ; Cowley, A. R.; Rogers, J. E.; Slagle, H. L.; Fleitz, P. A.; Drobizhev, M. ; Rebane, A.; Anderson, H. L. Org. Lett. 2005, 7, 5365-5368.

7. Chuchani, G.; Domiguez, R. M.; Rotinov, A.; Martin, I. J. Phys. Org, Chem. 1999, 12, 612-618.

Index of NMR spectra

NMR Spectrum : $\quad$ page

${ }^{1} \mathrm{H}$ NMR spectrum of $\mathbf{7 b}$

${ }^{13} \mathrm{C}$ NMR spectrum of $\mathbf{7 b}$

${ }^{1} \mathrm{H}$ NMR spectrum of $\mathbf{1 0}$

${ }^{13} \mathrm{C}$ NMR spectrum of $\mathbf{1 0}$

${ }^{1} \mathrm{H}$ NMR spectrum of $\mathbf{1 1}$

${ }^{13} \mathrm{C}$ NMR spectrum of $\mathbf{1 1}$

${ }^{1} \mathrm{H}$ NMR spectrum of $\mathbf{1 4}$

${ }^{13} \mathrm{C}$ NMR spectrum of $\mathbf{1 4}$

${ }^{1} \mathrm{H}$ NMR spectrum of $\mathbf{1 5}$

${ }^{13} \mathrm{C}$ NMR spectrum of $\mathbf{1 5}$

${ }^{1} \mathrm{H}$ NMR spectrum of $\mathbf{1 6}$

${ }^{13} \mathrm{C}$ NMR spectrum of $\mathbf{1 6}$
10

11

12

13

14

15

16

17

18

19

20

21 page

${ }^{1}$ H NMR spectrum of $\mathbf{1 8} \quad 22$

${ }^{13} \mathrm{C}$ NMR spectrum of $\mathbf{1 8} \quad \mathbf{2 3}$

${ }^{1} \mathrm{H}$ NMR spectrum of $\mathbf{1 9}$

${ }^{13} \mathrm{C}$ NMR spectrum of $\mathbf{1 9} \quad \mathbf{2 5}$

${ }^{1} \mathrm{H}$ NMR spectrum of $\mathbf{2 0} \quad \mathbf{2 6}$

${ }^{13} \mathrm{C}$ NMR spectrum of $\mathbf{2 0} \quad \mathbf{2 7}$

${ }^{1} \mathrm{H}$ NMR spectrum of $\mathbf{2 0}+\mathbf{2 1} \mathbf{2 8}$

${ }^{13} \mathrm{C}$ NMR spectrum of $\mathbf{2 0}+\mathbf{2 1} \mathbf{2 9}$

${ }^{2} \mathrm{H}$ NMR spectrum of $\mathbf{2 0}+\mathbf{2 1} 30$

${ }^{1} \mathrm{H}$ NMR spectrum of $\mathbf{2 4}$

${ }^{13} \mathrm{C}$ NMR spectrum of $\mathbf{2 4}$ 


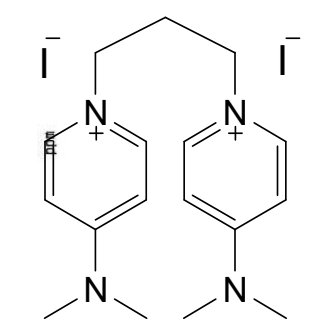

7b

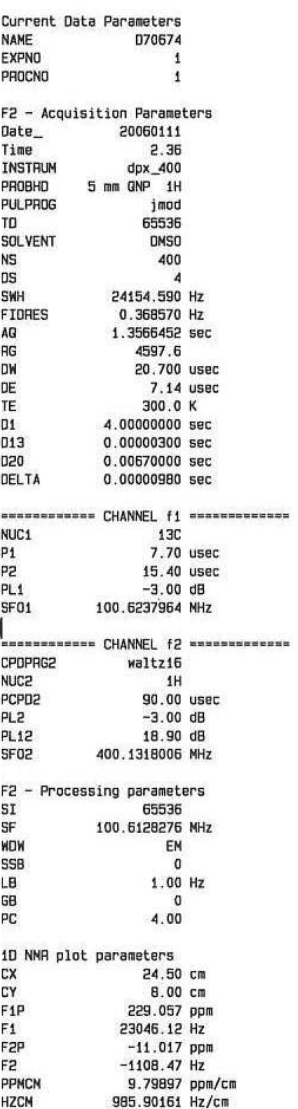




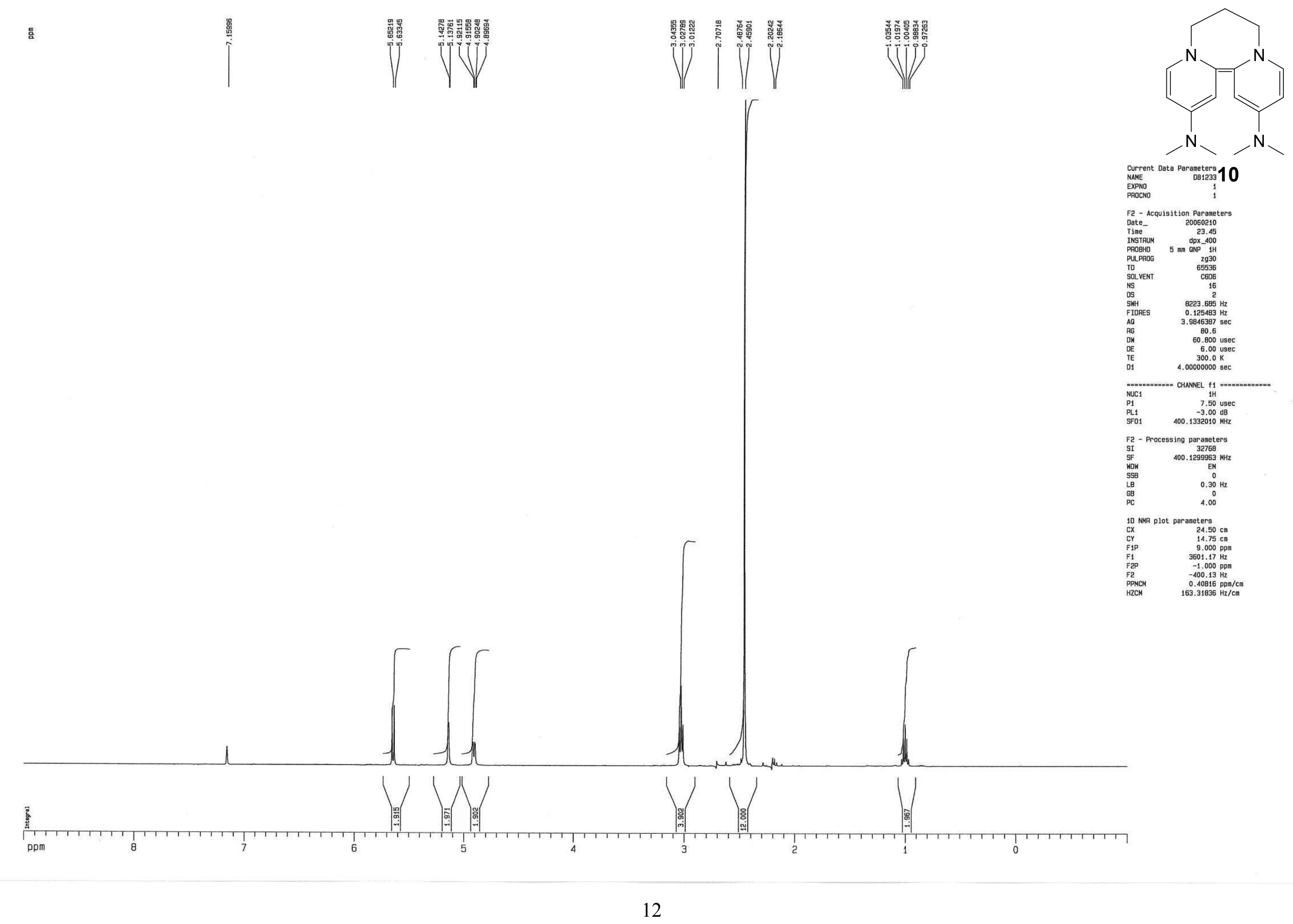



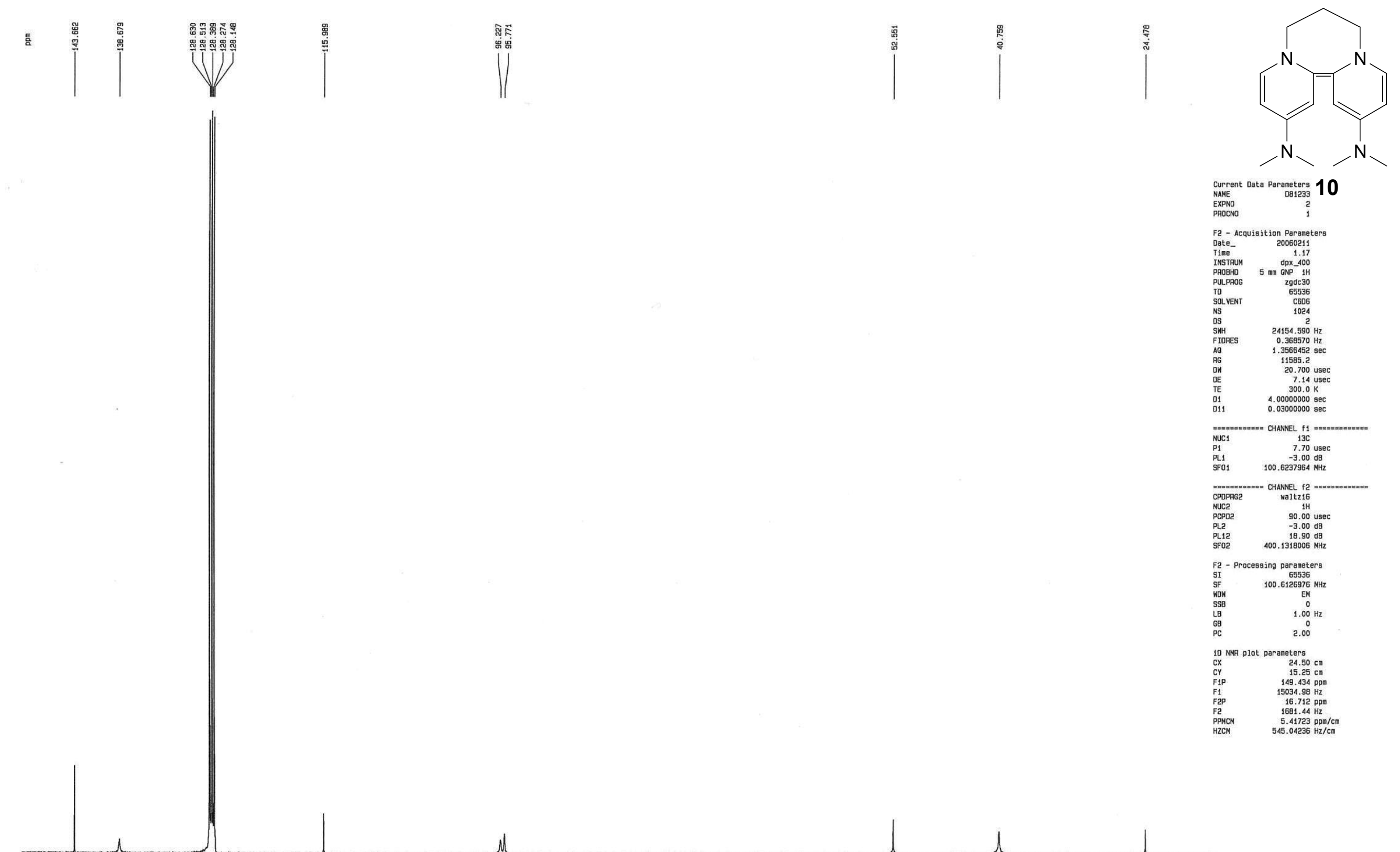


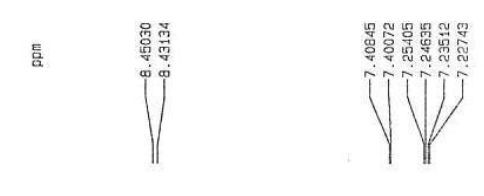

บบ

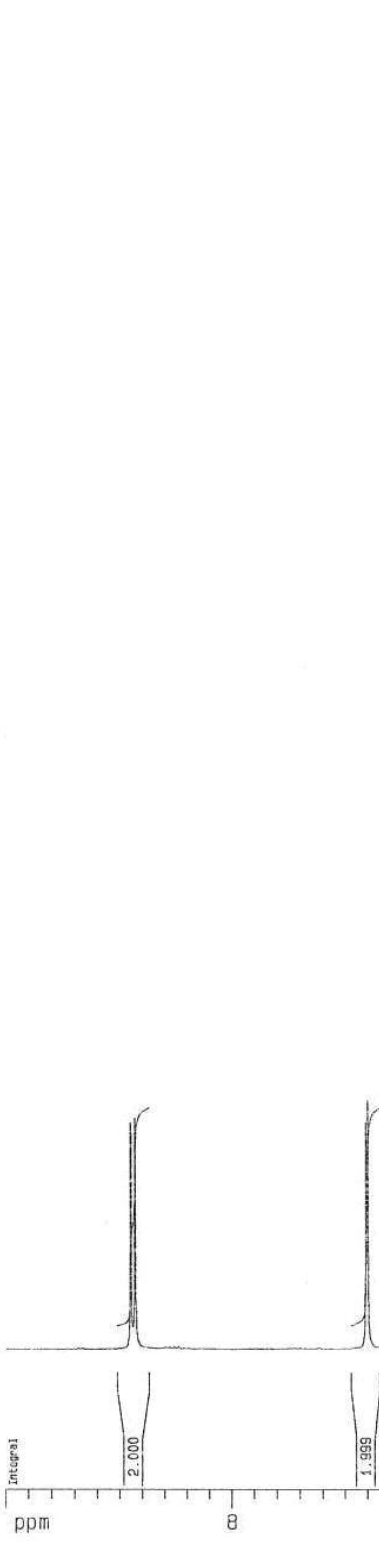

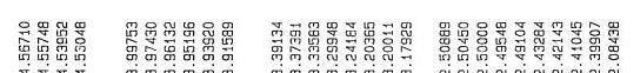

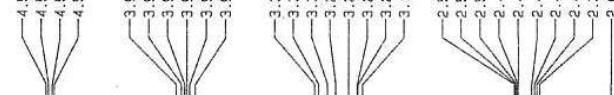

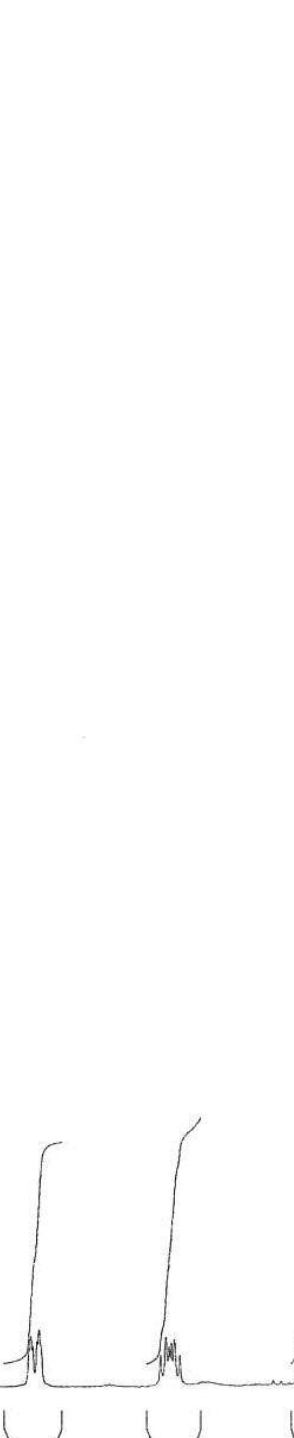

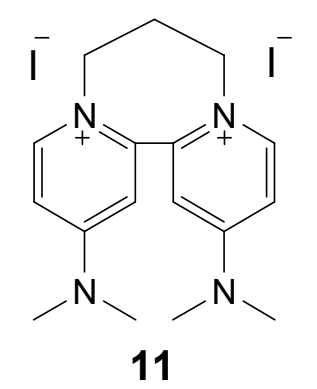

Current Data Par

EXPNO
PROCNO

1

Date-
Time $\quad \begin{aligned} 20000711 \\ 0.19\end{aligned}$

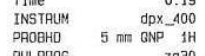

$\begin{aligned} 2930 \\ \text { PULPHOG }\end{aligned}$
TD
SOLVENT
DHSO

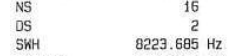

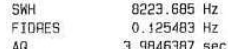

\begin{tabular}{lr} 
AB & $3.9846387 \mathrm{sec}$ \\
AG & 322.5 \\
OW & 60.000 usec \\
DE & 6.00 usec \\
\hline
\end{tabular}

$\begin{array}{ccc}\text { DE } & 6.00 \mathrm{usec} \\ \mathrm{TE} & 300 . \mathrm{K} \\ \mathrm{TE} & 0.10000000 \mathrm{sec} \\ 01 & \end{array}$

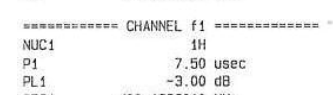

$\begin{array}{lr}\text { P1 } & 7.50 \text { usec } \\ \text { PL1 } & -3.0086 \\ \text { PF01 } & 400.19320000 \mathrm{NHz}\end{array}$

F2 - Processing paraneters
SI

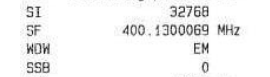

28
69

10 tiwn plot paraneters
CX $24.50 \mathrm{~cm}$

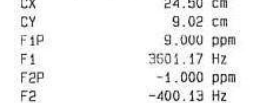

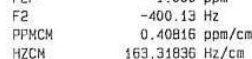



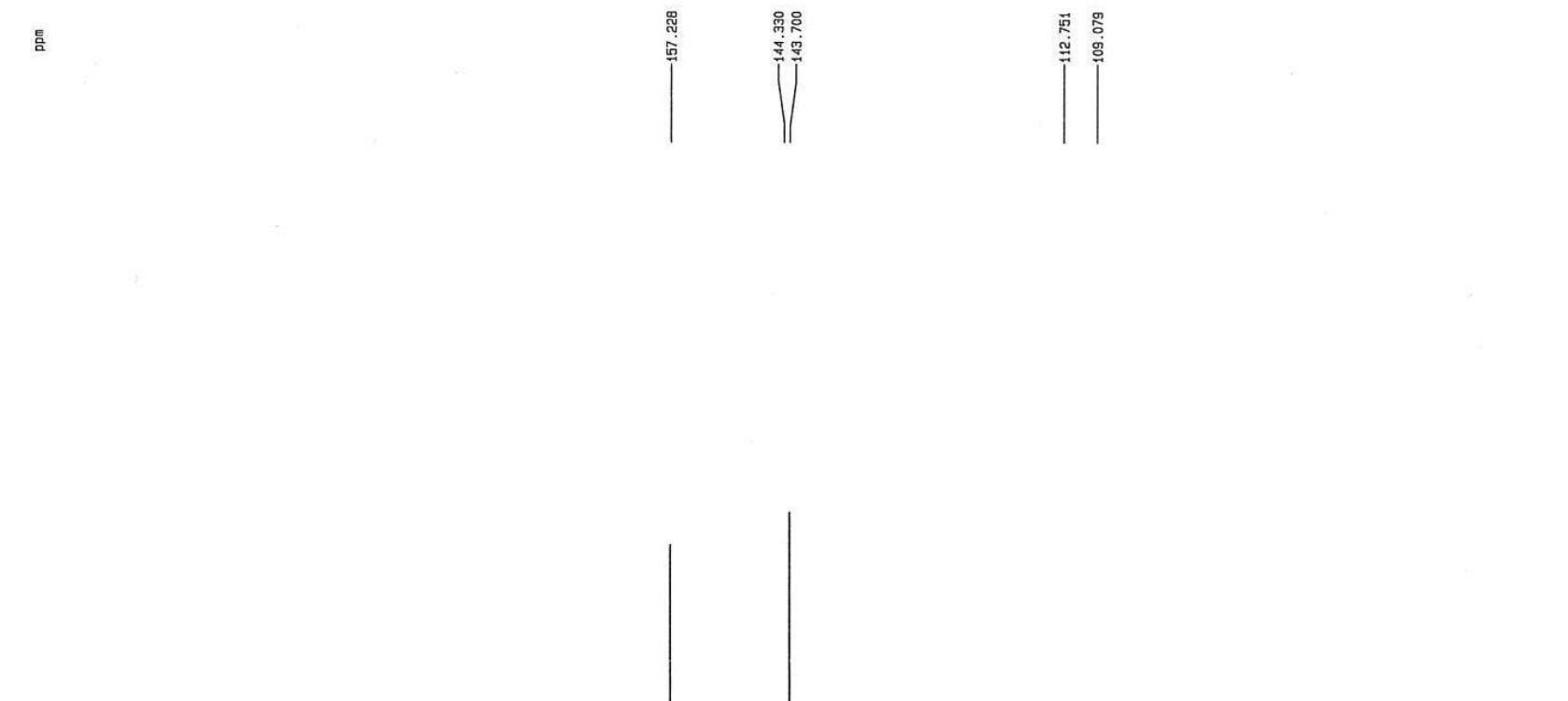

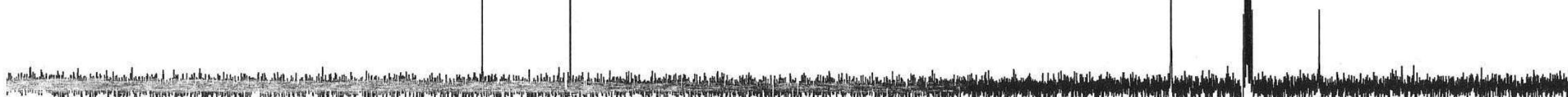

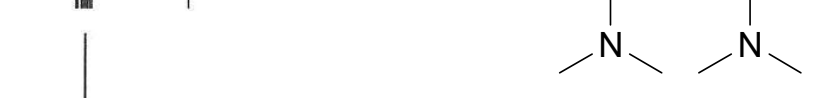

Current Data Parameter
NAME
EXPNo

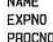

F2 - Acquisition Parameters

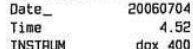

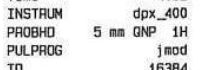

116384
TNSO
SOLVENT

$\begin{array}{lr}\text { NS } & 800 \\ \text { DS } & 4 \\ \text { SWH } & 24154.590 \mathrm{~Hz} \\ \text { FIDRES } & 1.474279 \mathrm{~Hz}\end{array}$

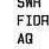

$A B$
AG
Oh
DE
$0 E$

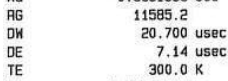

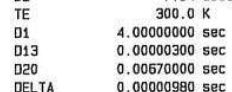

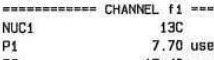

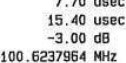

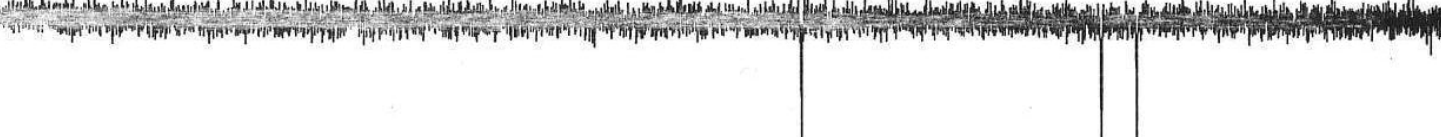
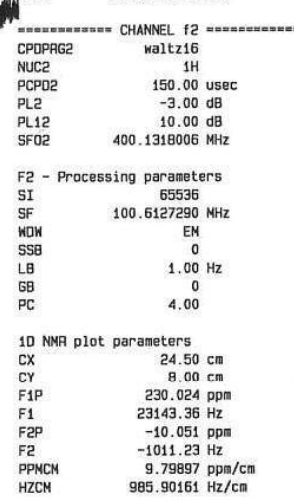

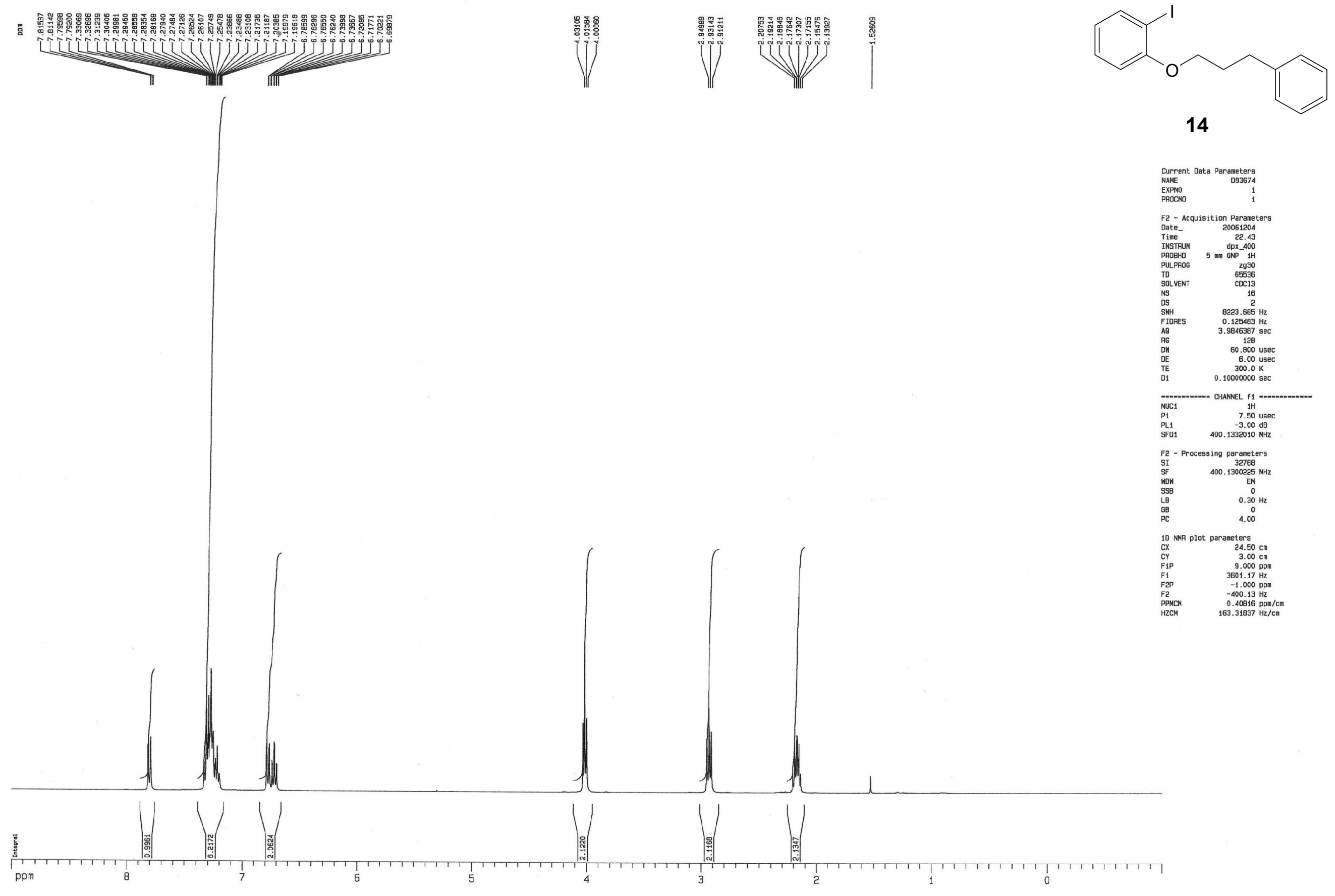


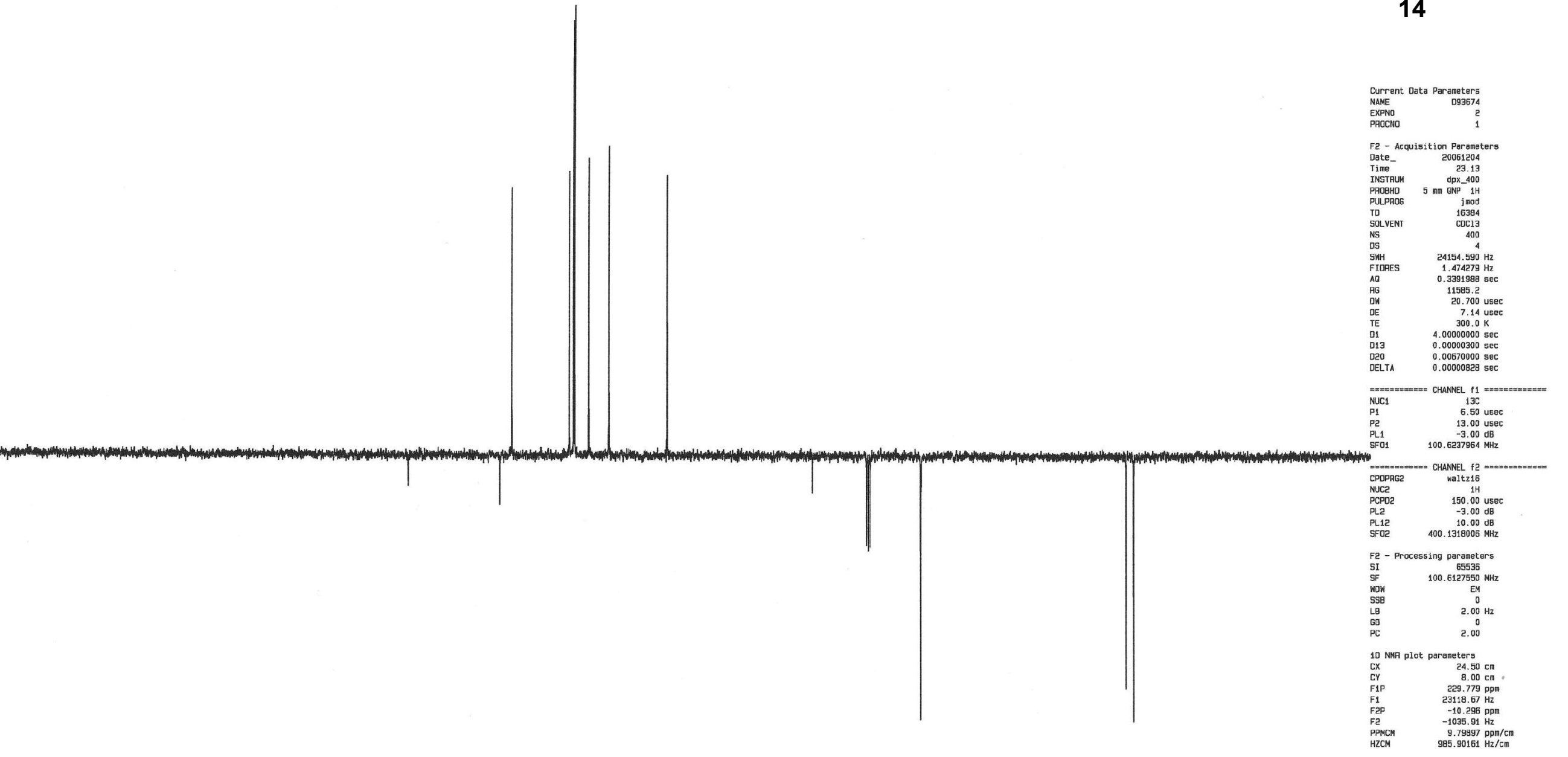



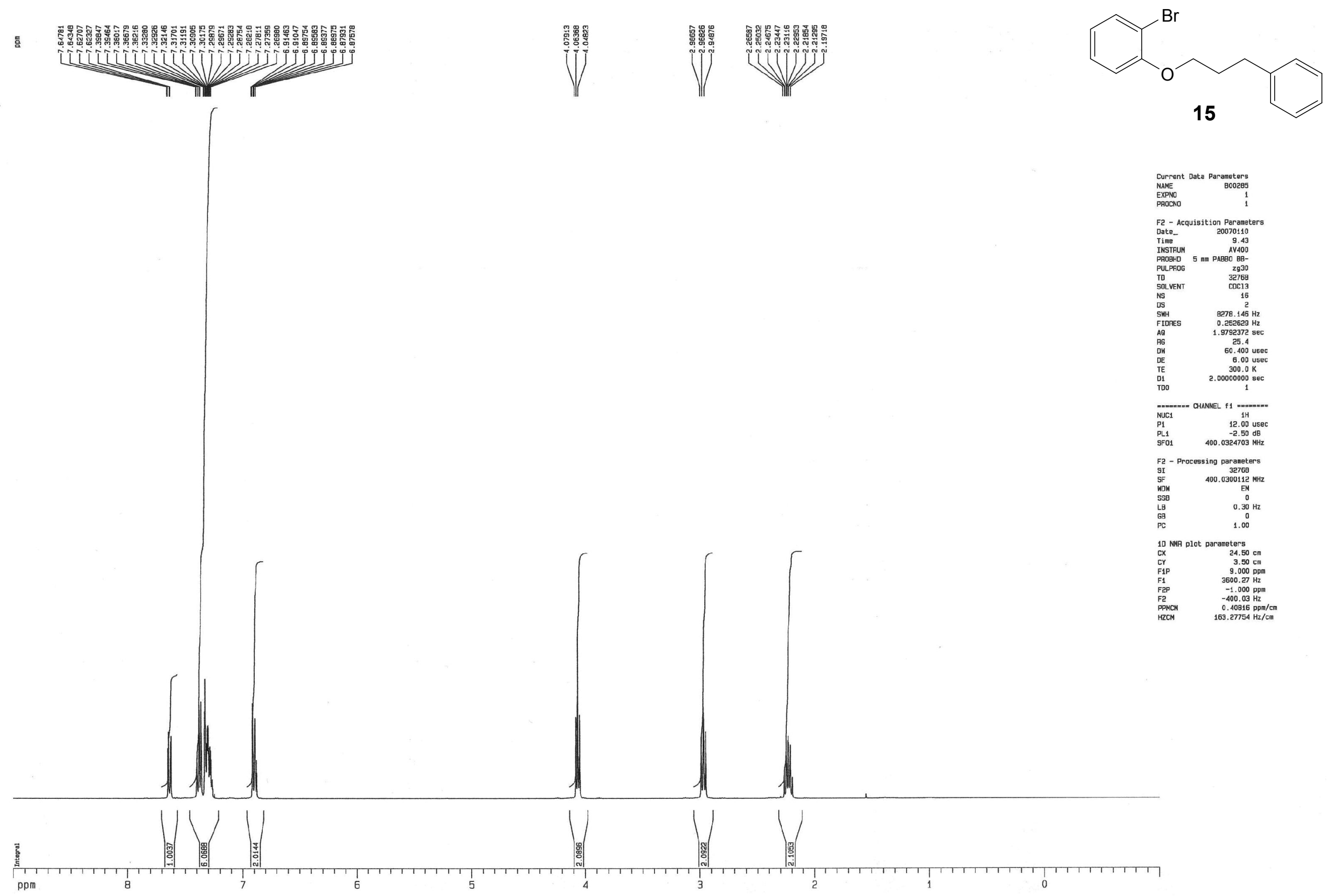


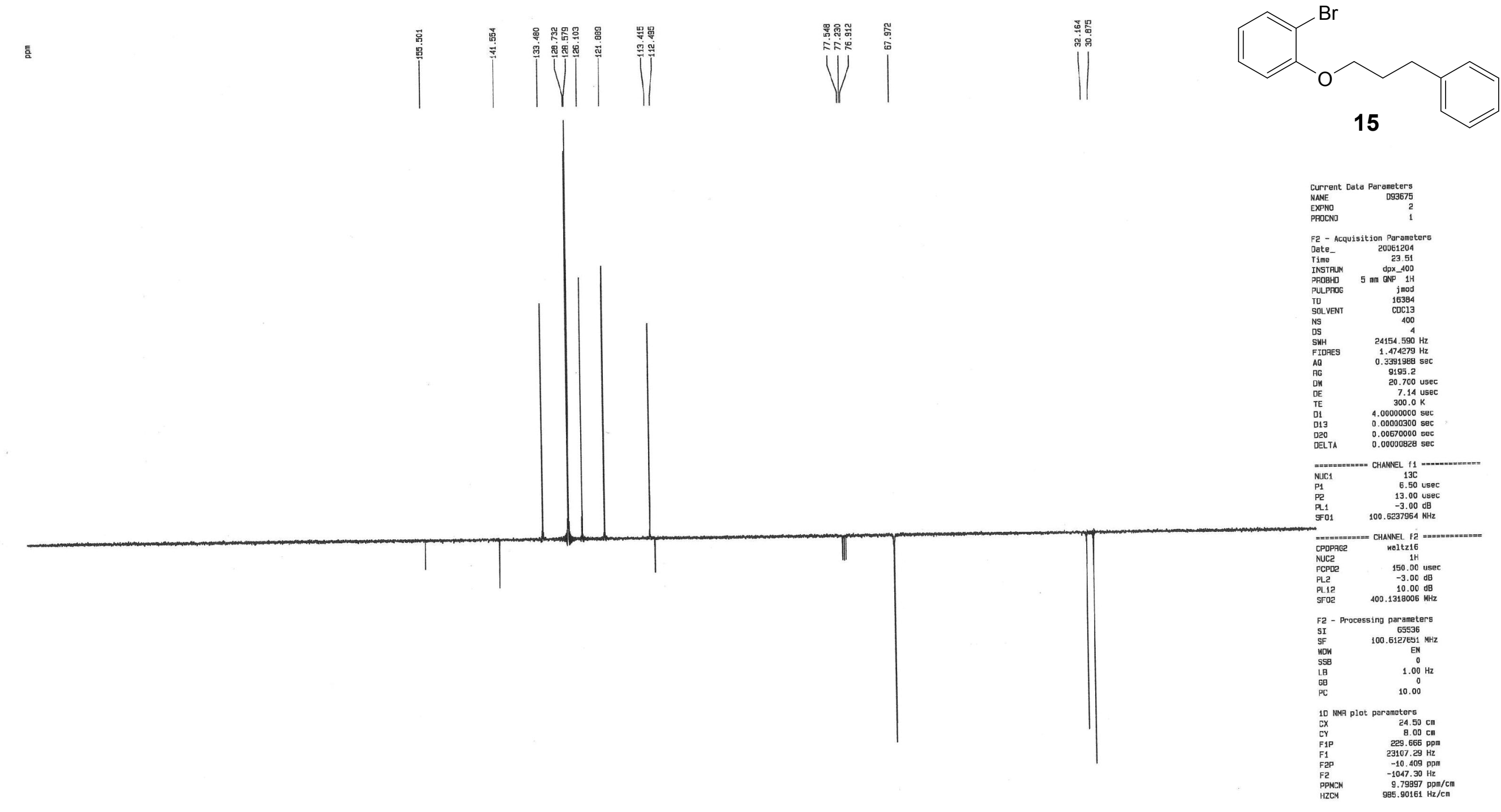



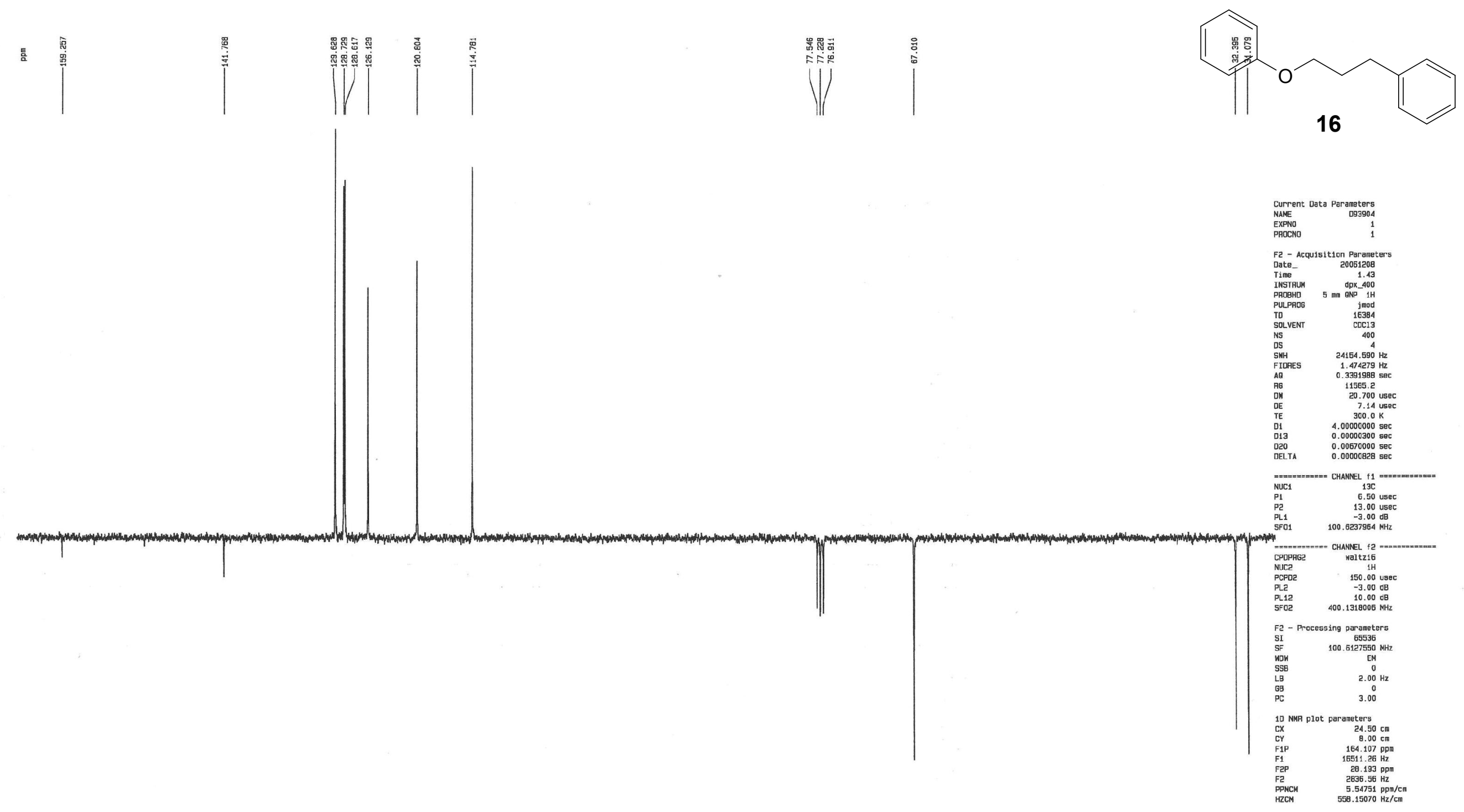

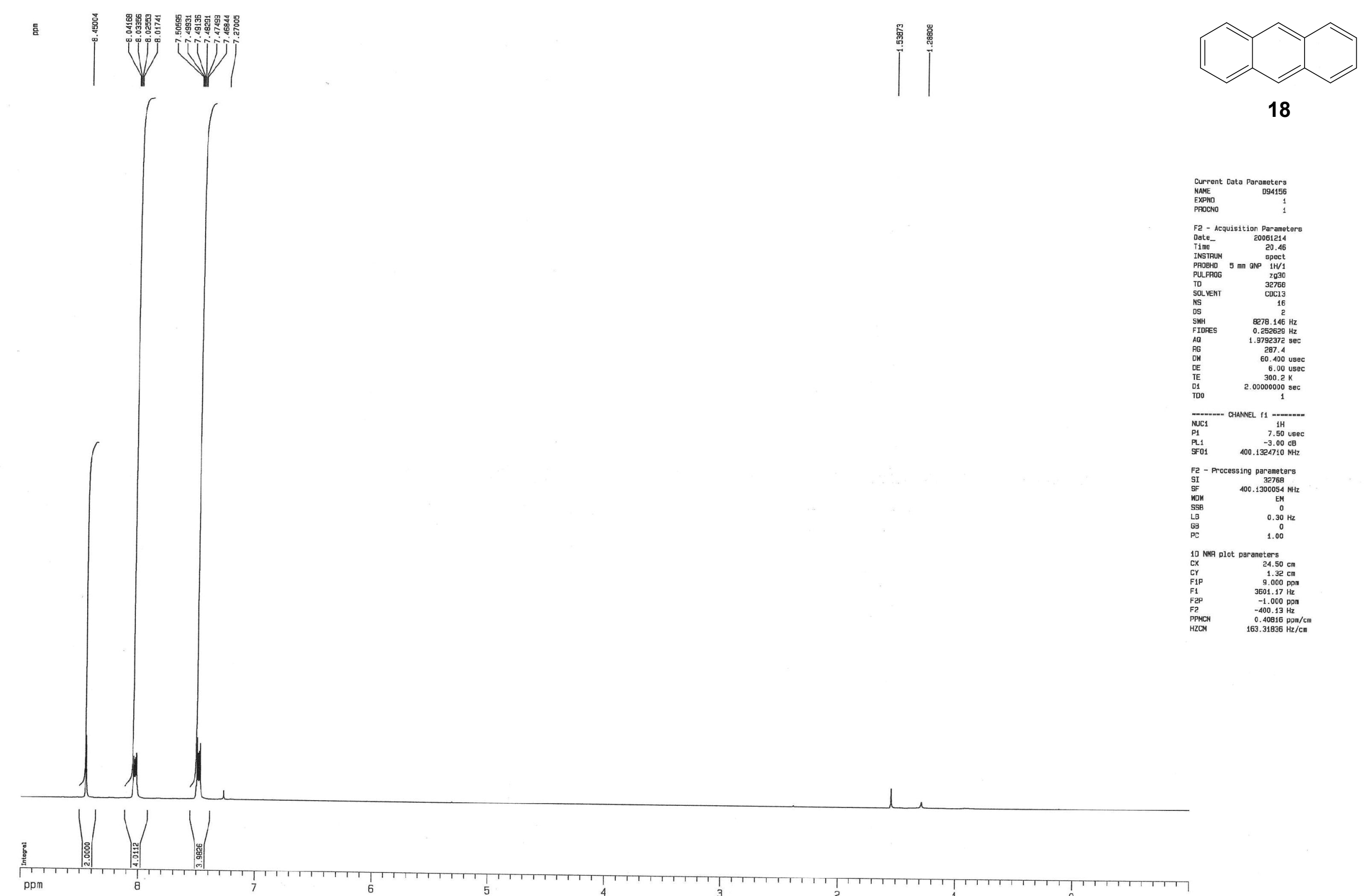
ppm $\frac{1}{7}$ 6 5 


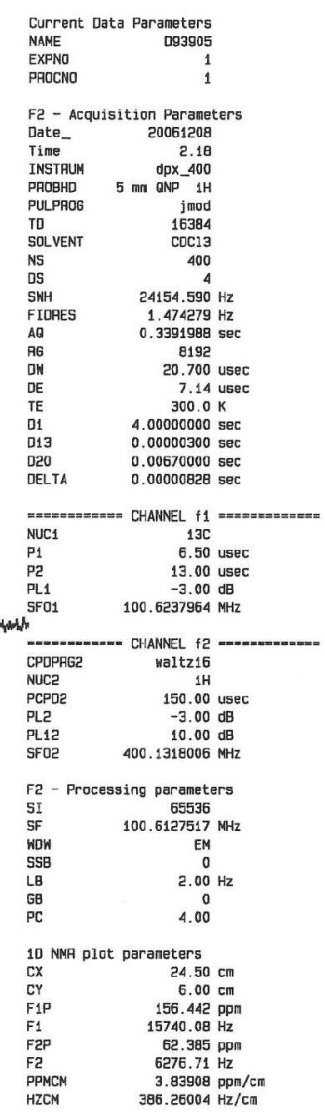




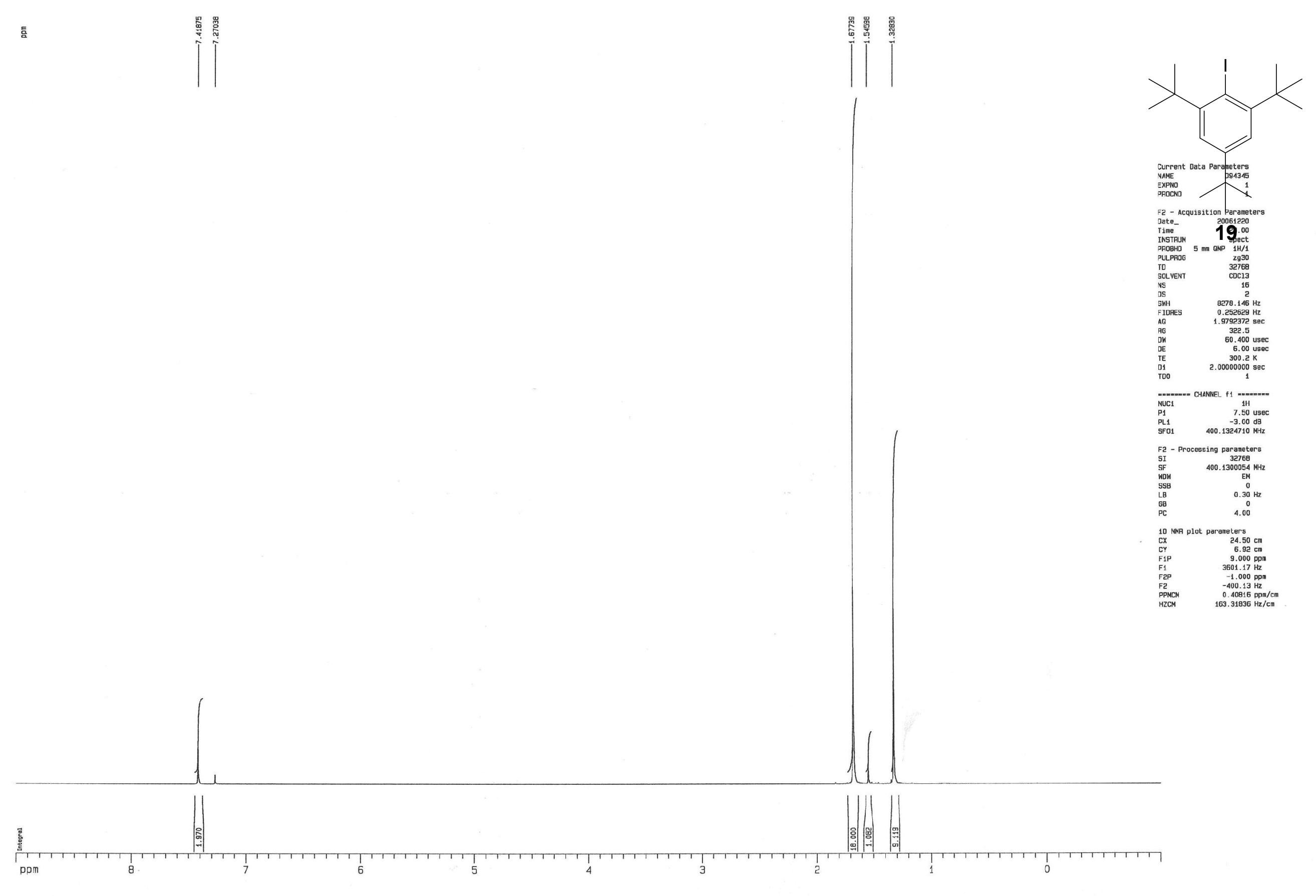




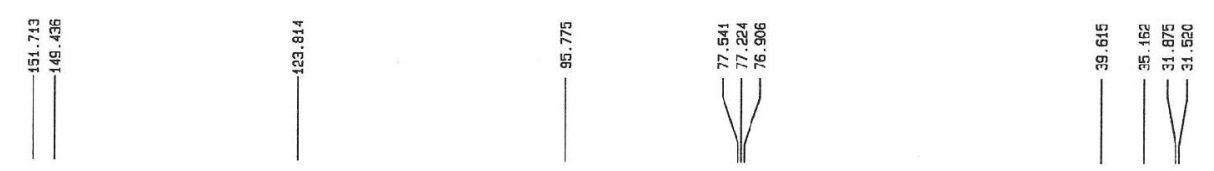
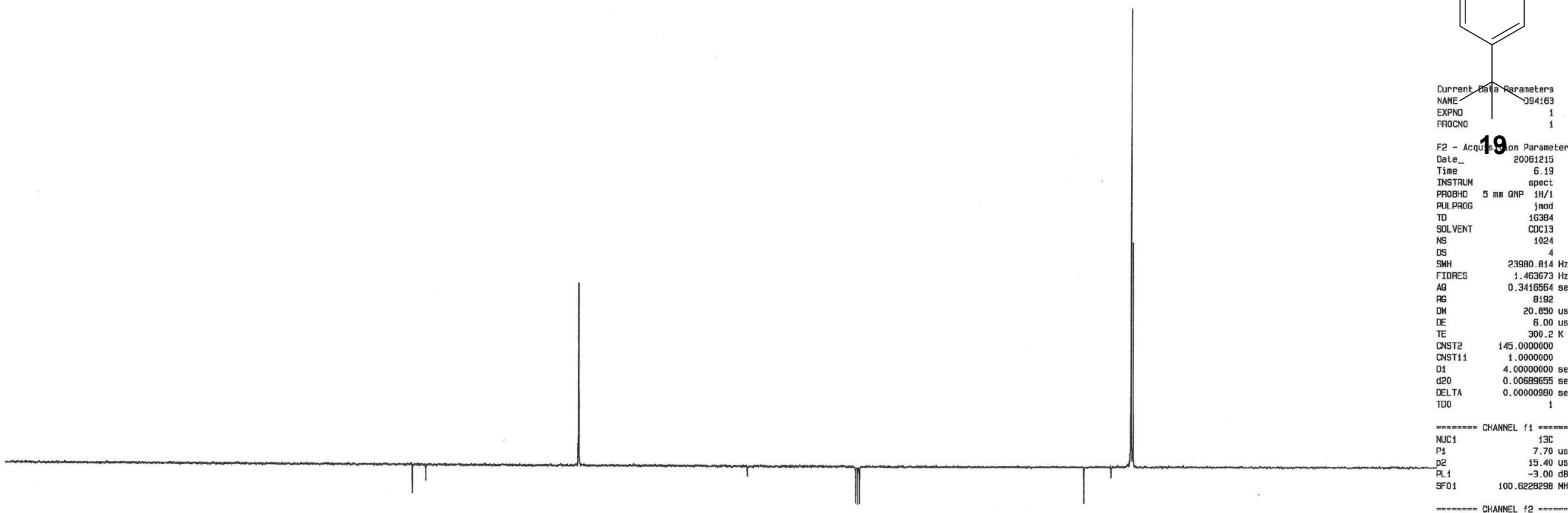

200611215
6 Tie.
spect
INSTRUM

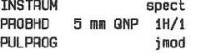

TO

1024
NS

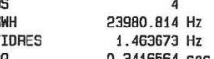

$0.3416564 \mathrm{sec}$
1092
20.850

$20.8500 \mathrm{use}$
$5.00 \mathrm{usec}$
$300.2 \mathrm{~K}$

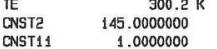

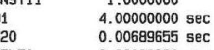

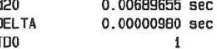

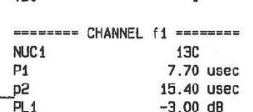

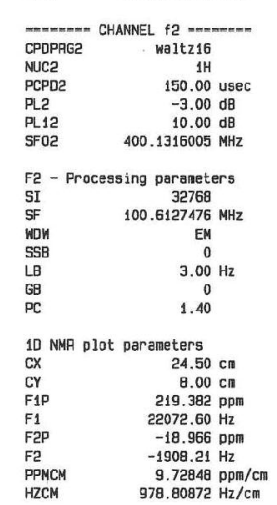


GA triterbutyl bzn

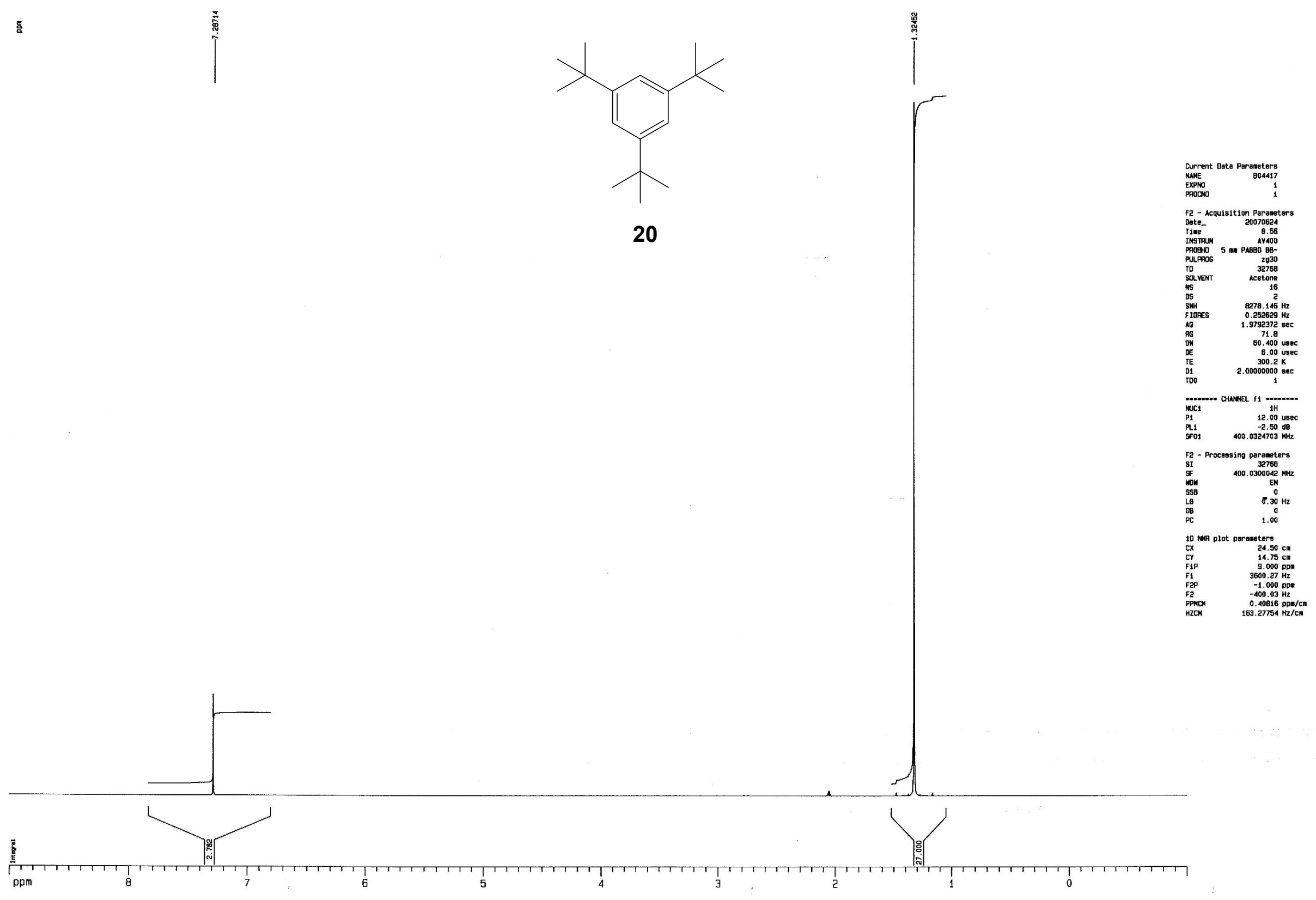




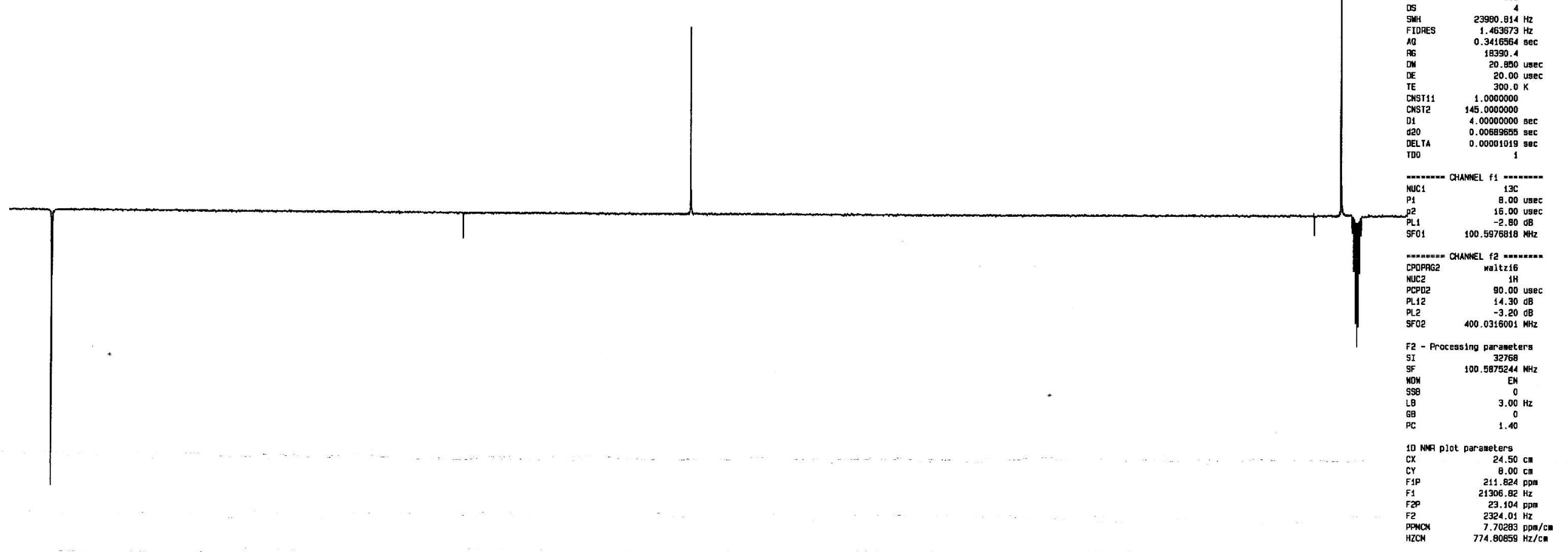


镸

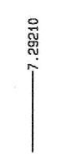

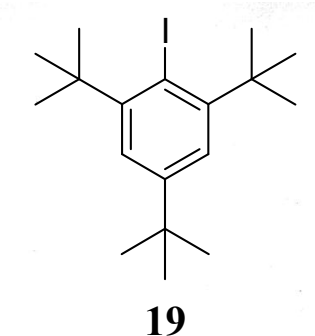
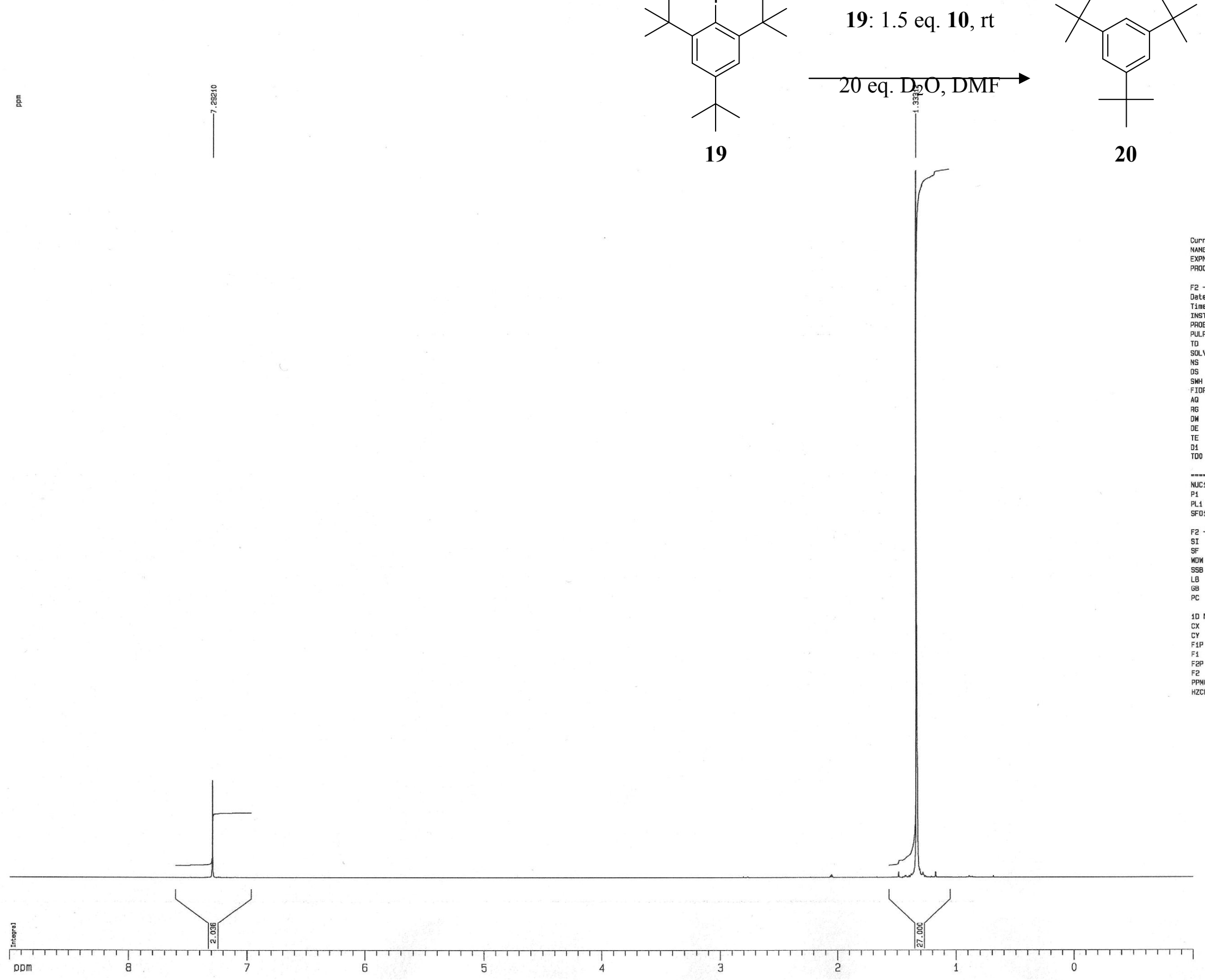

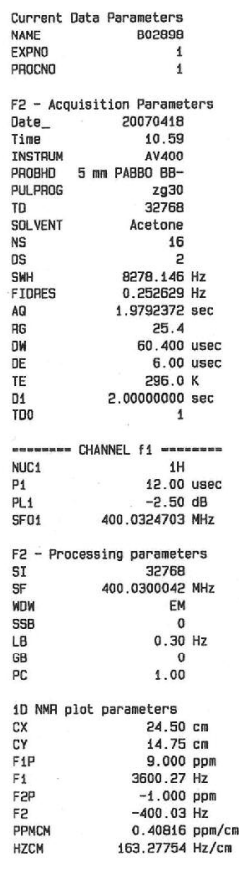


巨

1
19: 1.5 eq. 10 , rt

20 eq. $\mathrm{D}_{2} \mathrm{O}, \mathrm{DMF} \longrightarrow$

19

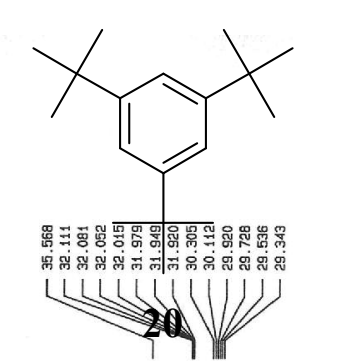

$+$
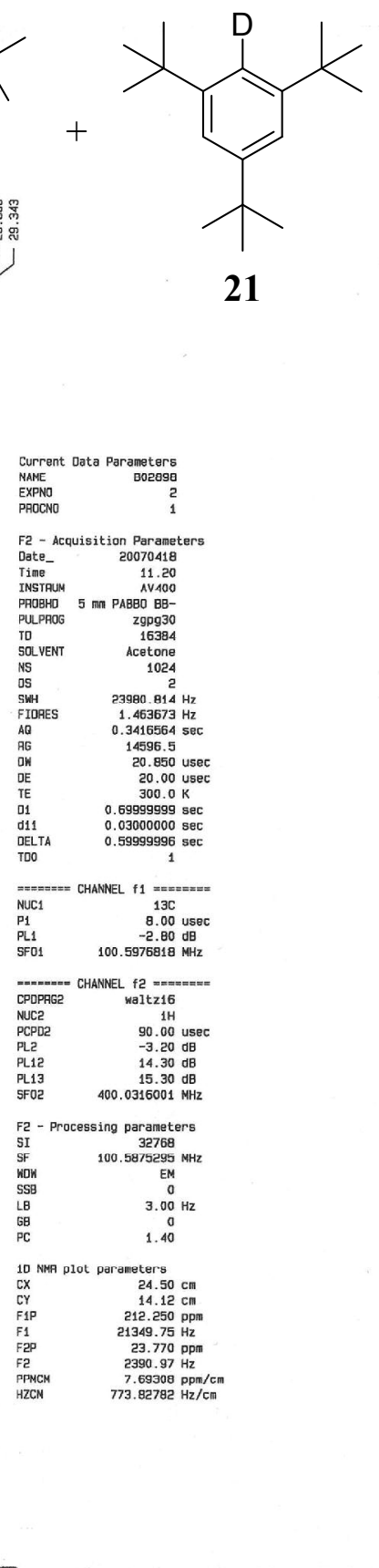

$\pi$

200

180

160

140

100

80

60

40 


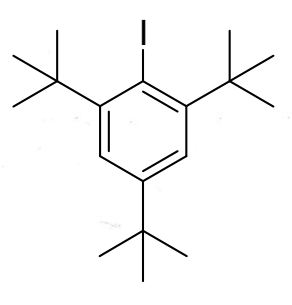

19
23: 1.5 eq. $14, \mathrm{rt}$

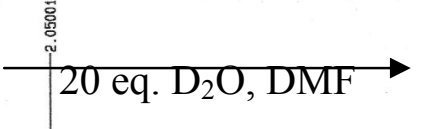
20 eq. $D_{2} \mathrm{O}, \mathrm{DMF}$

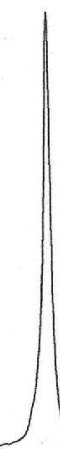

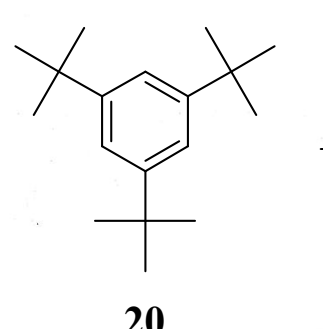

$+$
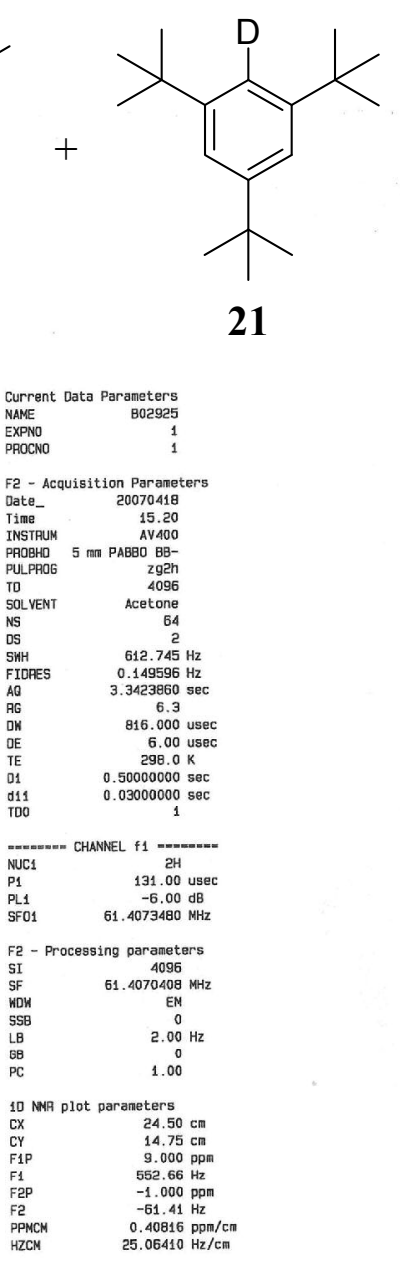


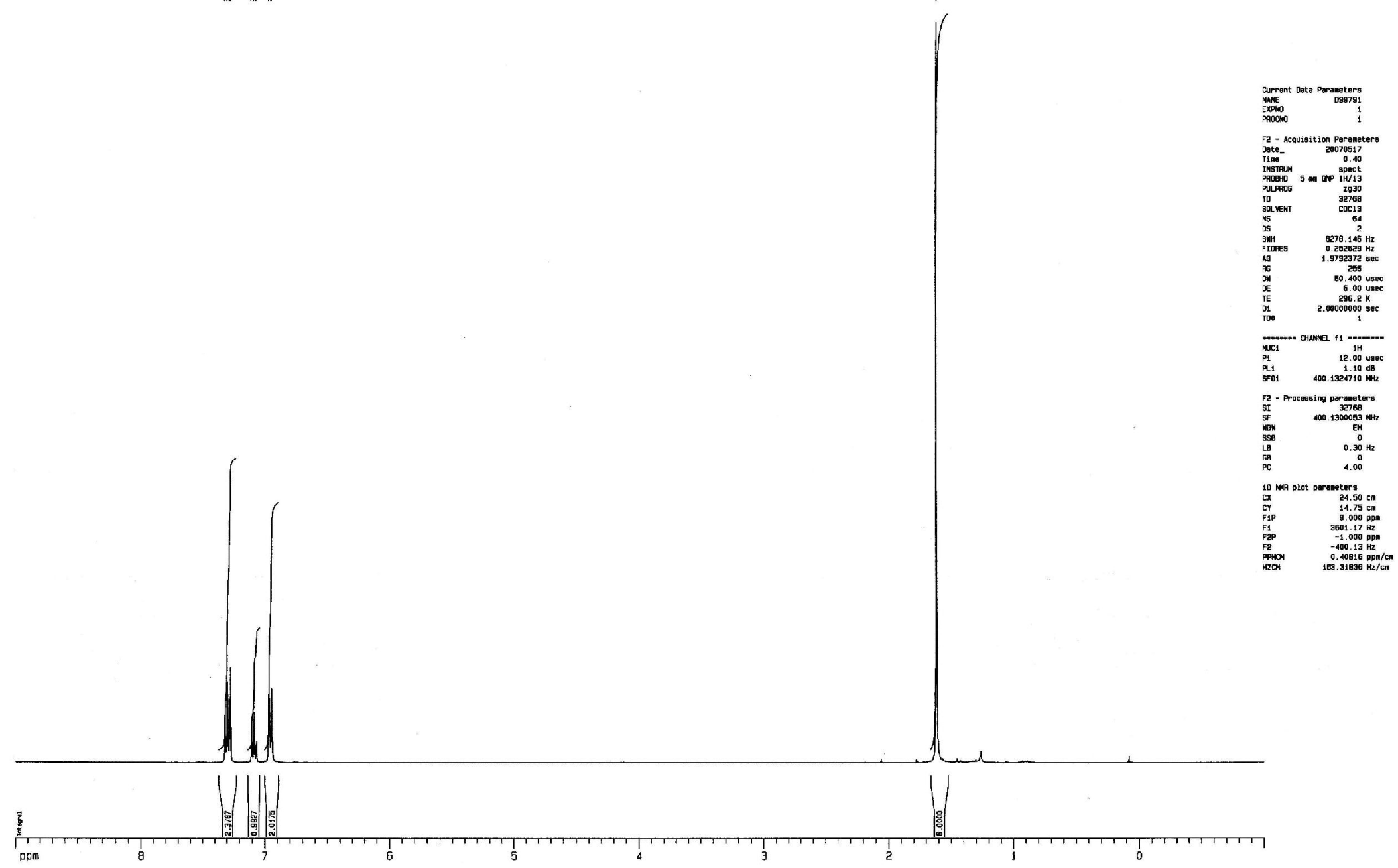



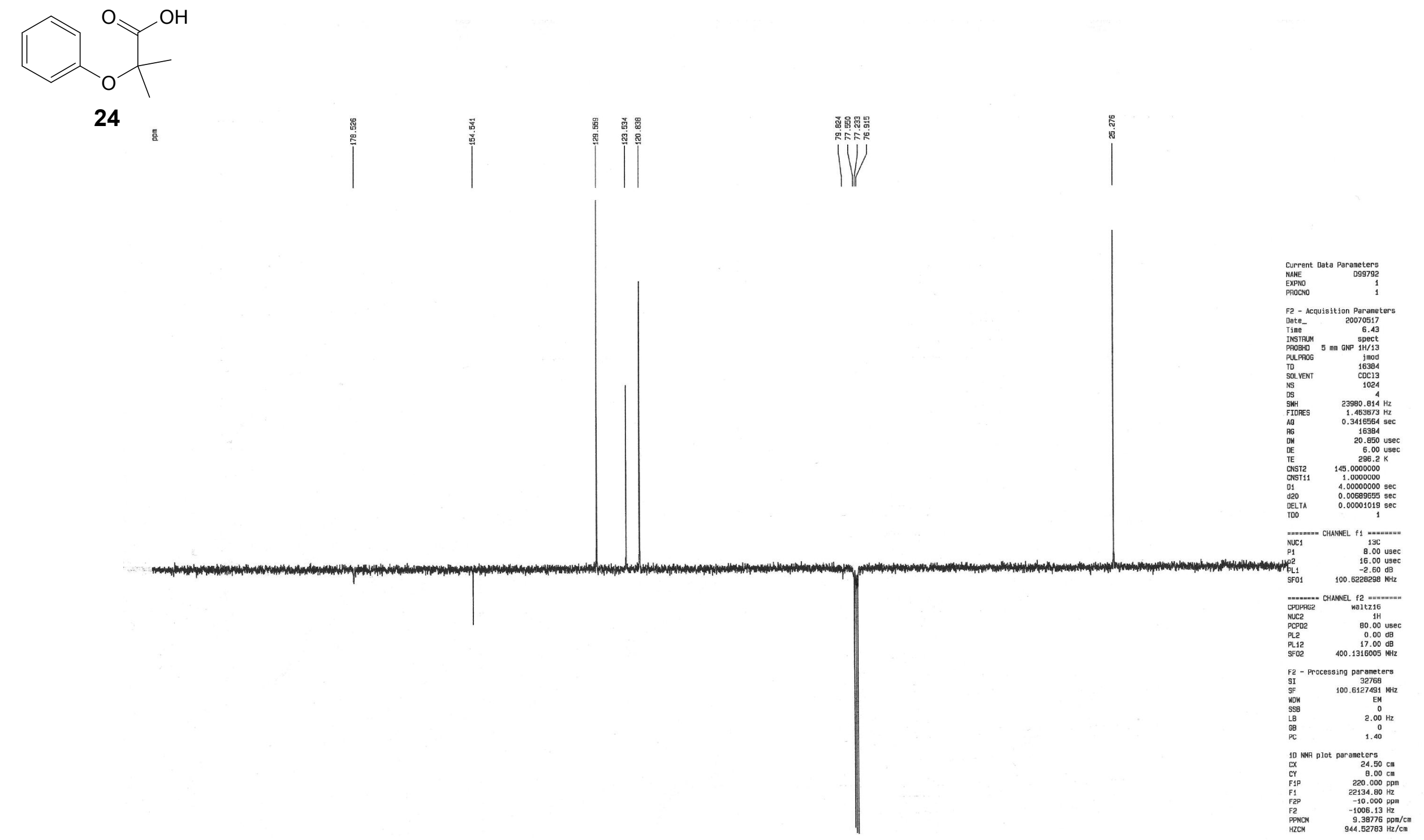